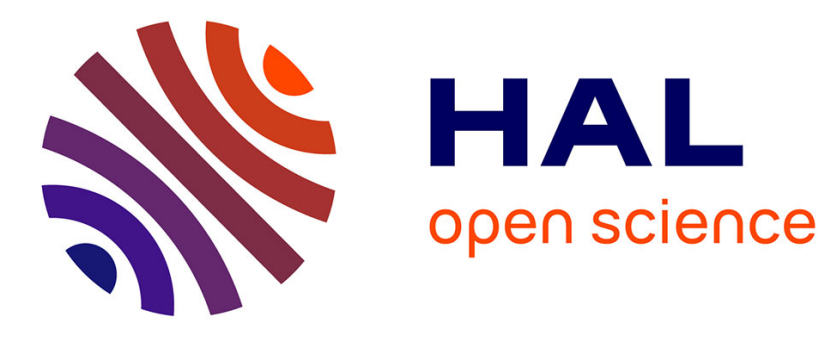

\title{
The Girsanov theorem without (so much) stochastic analysis
}

Antoine Lejay

\section{To cite this version:}

Antoine Lejay. The Girsanov theorem without (so much) stochastic analysis. Donati-Martin, Catherine; Lejay, Antoine; Rouault, Alain. Séminaire de Probabilités XLIX, 2215, Springer-Nature, 2018, 978-3-319-92419-9. 10.1007/978-3-319-92420-5_8. hal-01498129v3

\section{HAL Id: hal-01498129 \\ https://hal.inria.fr/hal-01498129v3}

Submitted on 22 Sep 2018

HAL is a multi-disciplinary open access archive for the deposit and dissemination of scientific research documents, whether they are published or not. The documents may come from teaching and research institutions in France or abroad, or from public or private research centers.
L'archive ouverte pluridisciplinaire HAL, est destinée au dépôt et à la diffusion de documents scientifiques de niveau recherche, publiés ou non, émanant des établissements d'enseignement et de recherche français ou étrangers, des laboratoires publics ou privés. 


\title{
The Girsanov theorem without (so much) stochastic analysis
}

\author{
Antoine Lejay*
}

June 13, 2018

\begin{abstract}
In this pedagogical note, we construct the semi-group associated to a stochastic differential equation with a constant diffusion and a Lipschitz drift by composing over small times the semi-groups generated respectively by the Brownian motion and the drift part. Similarly to the interpretation of the Feynman-Kac formula through the Trotter-Kato-Lie formula in which the exponential term appears naturally, we construct by doing so an approximation of the exponential weight of the Girsanov theorem. As this approach only relies on the basic properties of the Gaussian distribution, it provides an alternative explanation of the form of the Girsanov weights without referring to a change of measure nor on stochastic calculus.
\end{abstract}

Keywords: Girsanov theorem, Lie-Trotter-Kato formula, Feynman-Kac formula, Stochastic differential equation, Euler scheme, splitting scheme, flow, heat equation, Cameron-Martin theorem.

\section{Introduction}

This pedagogical paper aims at presenting the Girsanov theorem - a change of measure for the Brownian motion - using the point of view of operator analysis. We start from the sole knowledge of the Brownian distribution and its main properties. By doing so, stochastic calculus is avoided excepted for identifying the limit. Therefore, in a simplified context, we give an alternative proof of a result which is usually stated and proved using stochastic analysis and measure theory.

In 1944, R.H. Cameron and W.T. Martin proved the celebrated theorem on the change of the Wiener measure. They later extend it [9, 10].

*Université de Lorraine, IECL, UMR 7502, Vandœuvre-lès-Nancy, F-54600, France CNRS, IECL, UMR 7502, Vandœuvre-lès-Nancy, F-54600, France

Inria, Villers-lès-Nancy, F-54600, France

E-mail: Antoine.Lejay@univ-lorraine.fr 
Theorem 1 (R.H. Cameron \& W.T. Martin [11]). Let $F$ be a continuous functional on the space of continuous functions $\mathrm{C}([0,1] ; \mathbb{R})$ with respect to the uniform norm. Let $b$ be a continuous function in $\mathrm{C}([0,1] ; \mathbb{R})$ whose derivative $b^{\prime}$ has bounded variation. Then for a Wiener process (or a Brownian motion $1 \mathrm{~W}$,

$$
\mathbb{E}[F(\mathrm{~W})]=\mathbb{E}\left[F(\mathbf{W}+b) \exp \left(-\int_{0}^{1} b^{\prime}(s) \mathrm{dW}_{s}-\frac{1}{2} \int_{0}^{1}\left|b^{\prime}(s)\right|^{2} \mathrm{~d} s\right)\right] .
$$

Later in 1960, I.V. Girsanov states in [20] a variant of this theorem for solutions of stochastic differential equations. Even when the diffusivity $\sigma$ is constant, the drift is itself a non-linear functional of the Brownian motion.

Theorem 2 (I.V. Girsanov, [20]). Let $\mathrm{W}$ be a n-dimensional Wiener process on a probability space $(\Omega, \mathcal{F}, \mathbb{P})$ with respect to a filtration $\left(\mathcal{F}_{t}\right)_{t \geq 0}$. Let $\mathbf{X}$ be the solution on $(\Omega, \mathbb{P})$ to

$$
\mathbf{X}_{t}(\omega)=\mathbf{X}_{0}(\omega)+\int_{0}^{t} a(s, \omega) \mathrm{dW}(s, \omega)+\int_{0}^{t} b(s, \omega) \mathrm{d} s
$$

where $a$ is matrix valued, $b$ is vector valued, and

(A1) The applications $a$ and $b$ are measurable with respect to $(s, \omega) \in[0,1] \times \Omega$.

(A2) For each $t \geq 0$, a is $\mathcal{F}_{t}$-measurable.

(A3) Almost everywher 2 , $\int_{0}^{1}\|a(t, \omega)\|^{2} \mathrm{~d} t<+\infty$.

(A4) Almost everywhere, $\int_{0}^{1}|b(t, \omega)| \mathrm{d} t<+\infty$.

Let $\phi=\left(\phi^{1}, \ldots, \phi^{n}\right)$ be a vector-valued function on $[0,1] \times \Omega$ such that $(\mathrm{A} 1)$ (A3) are satisfied.

Let us set $\widetilde{\mathbb{P}}[\mathrm{d} \omega]=\exp \left(\mathrm{Z}_{0}^{1}(\phi, \omega)\right) \mathbb{P}[\mathrm{d} \omega]$ where

$$
\mathrm{Z}_{s}^{t}(\phi, \omega)=\int_{s}^{t} \phi^{i}(u, \omega) \delta_{i j} \mathrm{dW}^{j}(u, \omega)-\frac{1}{2} \int_{0}^{t}\left(\sum_{i=1}^{n} \phi^{i}(u, \omega)^{2}\right) \mathrm{d} u .
$$

Let us also set $\widetilde{\mathrm{W}}(t, \omega)=\mathrm{W}(t, \omega)-\int_{0}^{t} \phi(s, \omega) \mathrm{d}$ s. If $\widetilde{\mathbb{P}}[\Omega]=1$, then $\widetilde{\mathrm{W}}$ is a Wiener process with respect to $\left(\mathcal{F}_{t}\right)_{t \geq 0}$ on $(\Omega, \mathcal{F}, \widetilde{\mathbb{P}})$ and $(\mathrm{X}, \widetilde{\mathbb{P}})$ is solution to

$$
\mathbf{X}_{t}(\omega)=\mathbf{X}_{0}(\omega)+\int_{0}^{t} a(s, \omega) \mathrm{dW}_{s}(\omega)+\int_{0}^{t}(b(s, \omega)+a(s, \omega) \phi(s, \omega)) \mathrm{d} s .
$$

Soon after, these results were extended in many directions, for example to deal with semi-martingales (see e.g. [51]). The study of the weights for the change of

\footnotetext{
${ }^{1}$ In the original paper [11], the result is stated for $2^{-1 / 2} \mathrm{~W}$ and $2^{1 / 2} b$.

${ }^{2}$ The norm of a matrix is $\|a\|=\left(\sum_{i, j=1}^{n}\left|a_{i, j}^{2}\right|\right)^{1 / 2}$ while the norm of a vector is $\|a\|=$ $\left(\sum_{i=1}^{n}\left|a_{i}\right|^{2}\right)^{1 / 2}$.
} 
measures and their exponential nature gives rise to the theory of Doléans-Dade martingales 14,35 .

These theorems provide us with measures which are equivalent to the Wiener's one. The converse is also true [42]: Absolute continuity of Wiener or diffusions measures can only be reached by adding terms of bounded variation [42, 46].

The Cameron-Martin and Girsanov theorems have a profound meaning as well as a deep impact on modern stochastic calculus. For example, for example, they are one of the cornerstones of Malliavin calculus [6, 36, likewise a major tool in filtering, statistics of diffusion processes [33, 34], mathematical finance [26], ...

The Girsanov theorem has also been extended to some Gaussian processes, including the fractional Brownian motion 18, 24.

The Feynman-Kac formula related the Brownian motion with some PDEs. It involves a probabilistic representation with an exponential weight (see Section 9.1). The Feynman-Kac formula could be proved by many ways, including stochastic calculus from one side and the Trotter-Kato-Lie formula on the other side (see 17 , Chap. 3, Sect. 5] or [12 for a nice introduction to this subject, and [21] for a proof of the Feynman-Kac formula with this procedure).

To illustrate the latter approach, let us consider three linear matrix-valued equations

$$
\dot{X}=\mathfrak{A} X, \dot{Y}=\mathfrak{B} Y, \dot{R}=(\mathfrak{A}+\mathfrak{B}) R \text { with } X_{0}=Y_{0}=R_{0}=\mathrm{Id},
$$

where $\mathfrak{A}$ and $\mathfrak{B}$ are $d \times d$-matrices. These equations are easily solved by

$$
X_{t}=\exp (t \mathfrak{A}), Y_{t}=\exp (t \mathfrak{B}) \text { and } R_{t}=\exp (t(\mathfrak{A}+\mathfrak{B})) .
$$

The solutions $X, Y$ and $R$ satisfy the semi-group property: $X_{t+s}=X_{t} X_{s}$. If $\mathfrak{A} \mathfrak{B}=\mathfrak{B A}$, then $R_{t}=Y_{t} X_{t}$. This is no longer true in general. However, as shown first by S. Lie [32, the solution $R$ could be constructed from $X$ and $Y$ by the following limit procedure:

$$
R_{t}=\exp (t(\mathfrak{A}+\mathfrak{B}))=\lim _{n \rightarrow \infty} X_{t / n} Y_{t / n} X_{t / n} Y_{t / n} \cdots X_{t / n} Y_{t / n}=\lim _{n \rightarrow \infty}\left(e^{\mathfrak{A} t / n} e^{\mathfrak{B} t / n}\right)^{n} .
$$

H.F. Trotter [50] and T. Kato [28] have shown that this could be generalized for large families of linear unbounded operators $\mathfrak{A}$ and $\mathfrak{B}$. In this case, $\left(X_{t}\right)_{t \geq 0}$ and $\left(Y_{t}\right)_{t \geq 0}$ are families on linear operators with the semi-groups property.

On the space of continuous, bounded functions $\mathrm{C}_{\mathrm{b}}\left(\mathbb{R}^{d}, \mathbb{R}\right)$, consider the (scaled) Laplace operator $\mathfrak{A}=\frac{1}{2} \triangle$ and let $\mathfrak{B}$ be defined by $\mathfrak{B} f(x)=U(x) f(x)$ for any $f \in$ $\mathrm{C}\left(\mathbb{R}^{d}, \mathbb{R}\right)$, where the continuous function $U$ is called a potential. For a Brownian motion $\mathrm{B}, X_{t}=\mathbb{E}\left[f\left(x+\mathrm{B}_{t}\right)\right]$ whereas $Y_{t} f(x)=\exp (t U(x)) f(x)$ for any $f \in$ $\mathrm{C}_{\mathrm{b}}\left(\mathbb{R}^{d}, \mathbb{R}\right), x \in \mathbb{R}^{d}$ and $t \geq 0$. 
With the Trotter-Kato-Lie formula, we compose over short times the semigroup $X$ of the Laplace operator with the one $Y$ of the potential term. Using the Markov property of the Brownian motion, for any bounded, measurable function $f$,

$$
R_{t} f(x)=\lim _{n \rightarrow \infty} \mathbb{E}\left[\exp \left(\frac{t}{n} \sum_{i=0}^{n-1} U\left(x+\mathrm{B}_{i t / n}\right)\right) f\left(x+\mathrm{B}_{t}\right)\right] .
$$

With the right integrability conditions on $U$, we obtain in the limit an exponential representation of the Feynman-Kac formula [27], that is

$$
R_{t} f(x)=\mathbb{E}\left[\exp \left(\int_{0}^{t} U\left(x+\mathrm{B}_{s}\right) \mathrm{d} s\right) f\left(x+\mathrm{B}_{t}\right)\right]
$$

which gives a probabilistic representation to the PDE

$$
\partial_{t} R_{t} f(x)=\frac{1}{2} \triangle R_{t} f(x)+U(x) R_{t} f(x) \text { with } R_{0} f(x)=f(x) .
$$

The seminal derivation of the Feynman-Kac formula by M. Kac in 27] used an approximation of the Brownian motion by a random walk, which leads to an expression close to (1).

What happens now if we use for $\mathfrak{B}$ the first-order differential operator $b \nabla \cdot$ for a function $b$ ? This means that we consider giving a probabilistic representation of the semi-group $\left(R_{t}\right)_{t \geq 0}$ related to the PDE

$$
\partial_{t} R_{t} f(x)=\frac{1}{2} \triangle R_{t} f(x)+b(x) \nabla R_{t} f(x) \text { with } R_{0} f(x)=f(x) .
$$

Of course, a probabilistic representation is derived by letting $\mathrm{X}_{t}$ be the solution of the SDE $\mathbf{X}_{t}=x+\mathrm{B}_{t}+\int_{0}^{t} b\left(\mathbf{X}_{s}\right) \mathrm{d} s$ and $R_{t} f(x)=\mathbb{E}\left[f\left(\mathbf{X}_{t}\right)\right]$. With the Girsanov theorem (Theorem 2), $R_{t} f(x)=\mathbb{E}\left[\mathrm{Z}_{t} f\left(x+\mathrm{B}_{t}\right)\right]$ for the Girsanov weight $\mathrm{Z}$ given by (5) below.

The Feynman-Kac formula is commonly understood as a byproduct of the Trotter-Kato-Lie formula [22], at least in the community of mathematical physics in relation with the Schrödinger equation [40, 45]. The Trotter-Kato-Lie formula also offers a simple interpretation, leading to splitting procedures that provide explicit construction of numerical schemes (see e.g., [8, 23], among others).

Splitting schemes have been proposed to solve SDE ${ }^{3}$. Surprisingly enough, we found no trace where the Girsanov theorem is presented as a by-product of the Trotter-Kato-Lie formulat Yet its probabilistic interpretation is very simple: we

\footnotetext{
${ }^{3}$ In the domain of SDE, among others, the Ninomiya-Victoir scheme 41] relies on an astute way to compose the operators.

${ }^{4}$ In [31], R. Léandre gives an interpretation of the Girsanov formula and Malliavin calculus in terms of manipulation on semi-groups.
} 
combine Brownian evolution over a short time with a transport equation through the ODE $\dot{\mathbf{X}}=b(\mathrm{X})$ over a short time, and so on...

A heuristic argument shows how the exponential weight appears. For a continuous, bounded function $f$, the semi-group solution $\left(P_{t}\right)_{t \geq 0}$ such that $\partial_{t} P_{t} f(x)=$ $\frac{1}{2} \triangle P_{t} f(x)$ is given by $P_{t} f(x)=\mathbb{E}\left[f\left(x+\mathrm{B}_{t}\right)\right]$. The one $\left(Q_{t}\right)_{t \geq 0}$ that gives the solution to $\partial_{t} Q_{t} f(x)=b(x) \nabla Q_{t} f(x)$ is for $f \in \mathrm{C}_{\mathrm{b}}^{1}\left(\mathbb{R}^{d}, \mathbb{R}\right)$

$$
Q_{t} f(x)=f\left(\mathrm{Y}_{t}(x)\right)=f(x)+t b(x) \nabla f(x)+\mathrm{O}\left(t^{2}\right) \text { with } \dot{\mathrm{Y}}_{t}=b\left(\mathrm{Y}_{t}\right) \text { and } \mathrm{Y}_{0}=x
$$

Thus, in short time,

$$
\begin{aligned}
P_{t} Q_{t} f(x)=\mathbb{E}\left[f\left(x+\mathrm{B}_{t}\right)+t b(x\right. & \left.\left.+\mathrm{B}_{t}\right) \nabla f\left(x+\mathrm{B}_{t}\right)\right]+\mathrm{O}\left(t^{2}\right) \\
=\mathbb{E}[f(x & \left.\left.+\mathrm{B}_{t}\right)+t b(x) \nabla f\left(x+\mathrm{B}_{t}\right)\right] \\
& +\mathbb{E}\left[t\left(b\left(x+\mathrm{B}_{t}\right)-b(x) \nabla f\left(x+\mathrm{B}_{t}\right)\right)\right]+\mathrm{O}\left(t^{2}\right) .
\end{aligned}
$$

Since the Gaussian density $p(t, x)=\exp \left(-x^{2} / 2 t\right) / \sqrt{2 \pi t}$ satisfies $\partial_{x} p(t, x)=-\frac{x}{t} p(t, x)$, an integration by parts implies that ${ }^{5}$

$\mathbb{E}\left[\nabla f\left(x+\mathrm{B}_{t}\right)\right]=\int p(t, y) \partial_{y} f(x+y) \mathrm{d} y=\int \frac{y}{t} p(t, y) f(x+y) \mathrm{d} y=\mathbb{E}\left[\frac{\mathrm{B}_{t}}{t} f\left(x+\mathrm{B}_{t}\right)\right]$

so that

$$
\mathbb{E}\left[t b(x) \nabla f\left(x+\mathrm{B}_{t}\right)\right]=\mathbb{E}\left[\mathrm{B}_{t} b(x) f\left(x+\mathrm{B}_{t}\right)\right] .
$$

Since $b$ is Lipschitz, the term involving $b\left(x+\mathrm{B}_{t}\right)-b(x)$ in $(3)$ is considered as a higher order term, roughly of order $\mathrm{O}\left(t^{3 / 2}\right)$ since $\mathrm{B}_{t}$ is roughly of order $\mathrm{O}(\sqrt{t})$ and it is multiplied by $t$. Thus,

$$
P_{t} Q_{t} f(x)=\mathbb{E}\left[f\left(x+\mathrm{B}_{t}\right)+\mathrm{B}_{t} b(x) f\left(x+\mathrm{B}_{t}\right)\right]+\mathrm{O}\left(t^{3 / 2}\right) .
$$

Again owing to the regularity of the Brownian motion since $\left(\mathrm{B}_{t}^{2}-t\right)_{t \geq 0}$ is a martingale, we get

$$
P_{t} Q_{t} f(x)=\mathbb{E}\left[\exp \left(b(x) \mathrm{B}_{t}-\frac{t}{2} b(x)^{2}\right) f\left(x+\mathrm{B}_{t}\right)\right]+\mathrm{O}\left(t^{3 / 2}\right)
$$

to take into account the Taylor expansion of the exponential at order 2. The exponential weight is an approximation of the Girsanov weight in short time so that a similar analysis may be performed to show that $P_{t} Q_{t} f(x)=\mathbb{E}\left[\mathrm{Z}_{t} f(x+\right.$ $\left.\left.\mathrm{B}_{t}\right)\right]+\mathrm{O}\left(t^{3 / 2}\right)$.

\footnotetext{
${ }^{5}$ This is one of the central ideas of Malliavin calculus to express the expectation involving the derivative of a function as the expectation involving the function multiplied by a weight.
} 
We also see that limit of our approach, as it requires both $f$ to be $\mathrm{C}_{\mathrm{b}}^{1}\left(\mathbb{R}^{d}, \mathbb{R}\right)$ and $b$ to be Lipschitz. Such a restriction does not hold with the classical approach.

Using the above set of ideas, we then aim at proving rigorously the Girsanov theorem in a restricted setting, by considering only the solution of the stochastic differential equation

$$
\mathrm{X}_{t}=x+\mathrm{B}_{t}+\int_{0}^{t} b\left(\mathrm{X}_{s}\right) \mathrm{d} s
$$

for a drift $b \in \mathrm{C}_{\mathrm{b}}^{1}\left(\mathbb{R}^{d}, \mathbb{R}^{d}\right)$ and a $d$-dimensional Brownian motion on a probability space $(\Omega, \mathcal{F}, \mathbb{P})$. As a rule, we try to stuck on functional analysis arguments, and not on stochastic calculus to see how far we could go. When $X$ is the canonical process ${ }^{6}$, we show that for any $f \in \mathrm{C}_{\mathrm{b}}^{1}\left(\mathbb{R}^{d}, \mathbb{R}\right)$,

$$
\begin{aligned}
\widehat{\mathbb{E}}\left[f\left(\mathbf{X}_{t}\right)\right] & =\mathbb{E}\left[\mathbf{Z}_{t} f\left(\mathbf{X}_{t}\right)\right] \\
\text { with } \mathbf{Z}_{t} & =\exp \left(\int_{0}^{t} b\left(\mathbf{X}_{s}\right) \mathrm{dB}_{s}-\frac{1}{2} \int_{0}^{t}\left|b\left(\mathbf{X}_{s}\right)\right|^{2} \mathrm{~d} s\right) \\
& =1+\int_{0}^{t} \mathbf{Z}_{s} b\left(\mathbf{X}_{s}\right) \mathrm{dB}_{s},
\end{aligned}
$$

where $\widehat{\mathbb{E}}$ is the expectation of the distribution $\widehat{\mathbb{P}}$ of $X$, and $\mathbb{P}$ the one of the Brownian motion. It is only at the last stage that stochastic calculus is used, to give the expression for $\mathbf{Z}$ by passing to the limit. All the other arguments come from functional analysis or simple computations on the Gaussian density.

In the course of events, we obtain an upper bound for the weak rate of convergence of the Euler scheme with a drift coefficient of class $\mathrm{C}_{\mathrm{b}}^{1}\left(\mathbb{R}^{d}, \mathbb{R}^{d}\right)$, as well as some insight on the exponential nature of the Girsanov weight.

We actually gives two derivations. One is based on the Euler scheme and could be used without reference to the splitting procedure. It was used, with a different formulation, by G. Maruyama in 1954 to show the differentiability of the transition densities of SDE [37. This approach now seems to be part of the "folklore". The second one is an application of the Trotter-Kato-Lie using the heuristic given above. Both procedures complement each others and gives two alternative discretization of the Girsanov weights.

Our hypotheses are a priori more restrictive than the full Girsanov theorem. Actually,

- Using the Markov property of $\mathbf{Z}$ and $\mathbf{X}$, it is possible to show that

$$
\mathbb{E}\left[\mathrm{Z}_{t_{2}} f\left(\mathrm{X}_{t_{2}}\right) f\left(\mathrm{X}_{t_{1}}\right)\right]=\mathbb{E}\left[\mathbb{E}_{\mathrm{X}_{t_{1}}}\left[\mathrm{Z}_{t_{2}, t_{1}} f\left(\mathrm{X}_{t_{2}-t_{1}}\right)\right] \mathrm{Z}_{t_{1}} f\left(\mathrm{X}_{t_{1}}\right)\right]=\widehat{\mathbb{E}}\left[f\left(\mathrm{X}_{t_{2}}\right) f\left(\mathrm{X}_{t_{1}}\right)\right]
$$

for $0 \leq t_{1} \leq t_{2}$, and so on. A limiting argument shows that the transform is valid for functionals of the Brownian motion, not only for the marginal distribution.

\footnotetext{
${ }^{6}$ This is, $\mathrm{X}_{t}(\omega)=\omega(t)$ when the probability space $\Omega$ is $\mathrm{C}\left(\mathbb{R}_{+}, \mathbb{R}\right)$.
} 
- The restriction that $f \in \mathrm{C}_{\mathrm{b}}^{1}\left(\mathbb{R}^{d}, \mathbb{R}\right)$ in $(4)$ is easily removed by using a sequence of smooth approximations of a function $f$ which is only continuous.

- The Girsanov theorem assumes nothing on the regularity of the drift and requires only integrability condition. Here, the hypothesis that $b$ is $\mathrm{C}_{\mathrm{b}}^{1}\left(\mathbb{R}^{d}, \mathbb{R}^{d}\right)$ (or at least Lipschitz continuous) is crucial for our analysis. Yet any drift could be reached by regularizing the drift both in the PDE and the expression of the change of measure. However, non-anticipative functional drifts cannot be treated by this approach. We comment this further in Section 10.

- We use the independence of the increments of the Brownian motion as well as the explicit expression of its density (the heat or Gaussian kernel) and its derivative. Considering a diffusion coefficient $\sigma$ which is smooth enough, we could apply to approach to

$$
\mathbf{X}_{t}=x+\int_{0}^{t} \sigma\left(\mathbf{X}_{s}\right) \mathrm{dB}_{s},
$$

where the Markov property is used, as well as Gaussian controls over the density of the process and its derivatives, as well as expressions over the derivative of $\mathrm{X}_{t}$ using Malliavin calculus. It requires fine stochastic and analytic tools while our aim is to be as basic as possible, we use only the Brownian motion seen as a process with independent, Gaussian increments.

- Time could be added in $b$, provided that $b$ is uniformly Lipschitz continuous in space with respect to the time and uniformly bounded.

Finally, let us mention an alternative derivation of the Girsanov theorem that relies mostly on algebraic manipulations on the exponential weight [1]. This approach also avoids stochastic calculus as much as they can. Yet more sophisticated tools than ours are used.

Outline. The semi-groups are introduced in Section 2, The main estimates on elementary steps are given in Section 3 . The convergence of the semi-groups associated to the Euler scheme is shown in Section 4, while the ones for the splitting procedure is shown in Section 5. The limits of the weights are identified in Section 7, leading to our proof of the Girsanov theorem. The infinitesimal generator of the SDE with drift is identified in Section 8 by the sole use of functional analysis. At last, to complement our idea to avoid stochastic calculus, we present in Section 9 a representation of the Girsanov weights that does not involve stochastic integrals for some special form the of drift. Finally, we discuss in Section 10 the difference between SDEs and ODEs. The article ends with two appendices.

\section{Brownian motion and flows}

For a dimension $d$, let $\mathrm{B}$ be a $d$-dimensional Brownian motion on a probability space $(\Omega, \mathcal{F}, \mathbb{P})$. The drift $b$ we consider belongs to $\mathrm{C}_{\mathrm{b}}^{1}\left(\mathbb{R}^{d}, \mathbb{R}\right)$, the space of bounded, continuous functions with a bounded, continuous first order derivatives. 
We set for convenience $\mathcal{B}_{t, s}(x, \omega)=x+\mathrm{B}_{t}(\omega)-\mathrm{B}_{s}(\omega)$ for any $x \in \mathbb{R}^{d}, t \geq s \geq 0$ and $\omega \in \Omega$. This family $\left(\mathrm{B}_{t, x}(x, \omega)\right)_{t \geq s \geq 0}$ is called a random dynamical system. Clearly,

$$
\mathcal{B}_{t, s}\left(\mathcal{B}_{s, r}(x, \omega), \omega\right)=\mathcal{B}_{t, r}(x, \omega) \text { for any } t \geq s \geq r \geq 0 \text { and any } x \in \mathbb{R}^{d} .
$$

This property (7) is called the flow property.

For any $s \geq 0$, let us define $\left(\mathcal{X}_{t, s}(x, \omega)\right)_{t \geq s}$ as the unique solution to

$$
\begin{aligned}
\mathcal{X}_{t, s}(x, \omega) & =x+\mathrm{B}_{t}(\omega)-\mathrm{B}_{s}(\omega)+\int_{s}^{t} b\left(\mathcal{X}_{r, s}(x, \omega)\right) \mathrm{d} r \\
& =\mathcal{B}_{t, s}(x, \omega)+\int_{s}^{t} b\left(\mathcal{X}_{r, s}(x, \omega)\right) \mathrm{d} r, t \geq s .
\end{aligned}
$$

Actually, there is no need of a theory of stochastic differential equation for this, so that $\mathcal{X}_{\cdot, s}(x, \omega)$ could be defined pathwise for any Brownian path $\mathrm{B}(\omega)$ of the Brownian motion B.

Proposition 1. For every $\omega$ in $\Omega$ and any starting point $x \in \mathbb{R}^{d}$, there exists a unique solution $\mathcal{X}(x, \omega)$ to (8) defined on $[s,+\infty)$.

Proof. Let us consider $\mathcal{U}_{t, s}(x, \omega)=\mathcal{X}_{t, s}(x, \omega)-\mathcal{B}_{t, s}(x, \omega)$ and set $\beta(t, y, \omega)=$ $b\left(\mathcal{B}_{t, s}(x)+y\right)$ so that $b\left(\mathcal{X}_{t, s}(x, \omega)\right)=\beta\left(t, \mathcal{U}_{t, s}(x, \omega), \omega\right)$. This way, $\mathcal{X}_{\cdot, s}(x, \omega)$ is solution to (8) if and only if $\mathcal{U}_{\cdot, s}(x, \omega)$ is solution to

$$
\mathcal{U}_{t, s}(x, \omega)=\int_{s}^{t} \beta\left(r, \mathcal{U}_{r, s}(x, \omega), \omega\right) \mathrm{d} r, t \geq s .
$$

For any $s \geq 0, x \in \mathbb{R}^{d}$ and $\omega \in \Omega$, Eq. (9) has a unique solution, since $\beta$ is bounded in time and Lipschitz continuous in space. Then, (8) has necessarily a unique solution as the map which transforms $\mathcal{X}$ to $\mathcal{U}$ is one-to-one.

To simplify our notations, we drop from now any reference to the event $\omega \in \Omega$, which is implicit.

From the uniqueness of the solution to (8), the family $\left(\mathcal{X}_{t, s}\right)_{t \geq s \geq 0}$ satisfies the flow property:

$$
\mathcal{X}_{t, s}\left(\mathcal{X}_{s, r}(x)\right)=\mathcal{X}_{t, s}(x) \text { for } 0 \leq r \leq s \leq t .
$$

For $x \in \mathbb{R}^{d}$, we also consider the solution to the one-dimensional ODE

$$
\mathcal{Y}_{t, r}(x)=x+\int_{r}^{t} b\left(\mathcal{Y}_{s, r}(x)\right) \mathrm{d} s, t \geq r .
$$

This family also satisfies the flow property: $\mathcal{Y}_{t, r}(x)=\mathcal{Y}_{t, s}\left(\mathcal{Y}_{s, r}(x)\right)$ for any $0 \leq$ $r \leq s \leq t$ and any $x \in \mathbb{R}^{d}$. As $b$ is time-homogeneous, $\mathcal{Y}_{t, s}(x)=\mathcal{Y}_{t-s}(x)$ for any $0 \leq s \leq t$. 
Let us introduce several families of linear operators on $\mathrm{C}_{\mathrm{b}}\left(\mathbb{R}^{d}, \mathbb{R}\right)$ to $\mathrm{C}_{\mathrm{b}}\left(\mathbb{R}^{d}, \mathbb{R}\right)$ defined by

$$
\begin{aligned}
X_{s, t} g(x) & =\mathbb{E}\left[g\left(\mathcal{X}_{t, s}(x)\right)\right], \\
P_{s, t} g(x) & =\mathbb{E}\left[g\left(\mathcal{B}_{t, s}(x)\right)\right], \\
Q_{s, t} g(x) & =g\left(\mathcal{Y}_{t, s}(x)\right), \\
V_{s, t} g(x) & =\mathbb{E}\left[\left(1+b(x)\left(\mathrm{B}_{t}-\mathrm{B}_{s}\right)\right) g\left(\mathcal{B}_{t, s}(x)\right)\right], \\
E_{s, t} g(x) & =\mathbb{E}\left[g\left(\mathcal{B}_{t, s}(x)+(t-s) b(x)\right)\right],
\end{aligned}
$$

for $g \in \mathrm{C}_{\mathrm{b}}\left(\mathbb{R}^{d}, \mathbb{R}\right)$ and any $0 \leq s \leq t$.

The time indices of $X$ have been inverted with respect to the ones of $\mathcal{X}$. The same convention holds for the other operators. The reason is the following: as the increments $\mathrm{B}_{t}-\mathrm{B}_{s}$ and $\mathrm{B}_{s}-\mathrm{B}_{r}$ are independent,

$$
\mathbb{E}\left[g\left(\mathcal{X}_{t, s}\left(\mathcal{X}_{r, s}(x)\right)\right]=\mathbb{E}\left[X_{s, t} g\left(\mathcal{X}_{r, s}(x)\right)\right]=X_{r, s} X_{s, t} g(x) .\right.
$$

Acting the same for the other operators,

$$
X_{r, s} X_{s, t}=X_{r, t}, Q_{r, s} Q_{s, t}=Q_{r, t} \text { and } P_{r, s} P_{s, t}=P_{r, t} .
$$

Besides, $\mathcal{X}_{s, s}(x)=x$ so that $X_{s, s}=$ Id. The same holds for the pairs $(\mathcal{Y}, Q)$ and $(\mathcal{B}, P)$. This means that $\left(X_{r, t}\right)_{0 \leq r \leq t}, \quad\left(Q_{r, t}\right)_{0 \leq r \leq t}$ and $\left(P_{r, t}\right)_{0 \leq r \leq t}$ are nonhomogeneous semi-groups 7 . Their infinitesimal generators are

$$
\mathfrak{B}:=b \nabla \text { for }\left(Q_{r, t}\right)_{0 \leq r \leq t}, \quad \mathfrak{L}:=\frac{1}{2} \triangle \text { for }\left(P_{r, t}\right)_{0 \leq r \leq t} \text { and } \mathfrak{A}:=\mathfrak{L}+\mathfrak{B} \text { for }\left(X_{r, t}\right)_{0 \leq r \leq t}
$$

with the appropriate domains (see Section 8).

\section{Some estimates}

The next lemma is central for our analysis. Its proof is immediate so that we skip it.

Lemma 1. For bounded, linear operators $A_{i}$ and $B_{i}, i=1, \ldots, n$,

$$
\prod_{i=1}^{n}\left(A_{i}+B_{i}\right)-\prod_{i=1}^{n} A_{i}=\sum_{j=1}^{n}\left(\prod_{i=1}^{j-1}\left(A_{i}+B_{i}\right)\right) B_{j}\left(\prod_{i=j+1}^{n} A_{i}\right) .
$$

Actually, such a lemma just follows from algebraic manipulations which are valid in any Banach algebra. In the context of semi-group, it can be seen as a discrete version of the perturbation formula [17, 43 which is central for the analysis of semigroups. This perturbation is also related to the so-called parametrix method for constructing densities (see e.g., 4 for an interpretation in the stochastic context).

\footnotetext{
${ }^{7}$ These semi-groups are actually time-homogeneous. We however found it more convenient to keep the time dependence for our purpose.
} 


\subsection{Differentiability of the flows}

We recall here a classical result about the differentability of the flow. A formal proof is given for the sake of clarity.

To simplify the notations, we set

$$
\beta=\|\nabla b\|_{\infty}
$$

Proposition 2. For any $0 \leq s \leq t$, the maps $x \mapsto \mathcal{Y}_{t, s}(x)$ and $x \mapsto \mathcal{X}_{t, s}(x)$ are differentiable. Besides,

$$
\left\|\nabla \mathcal{Y}_{t, s}(x)\right\| \leq \exp ((t-s) \beta) \text { and }\left\|\nabla \mathcal{X}_{t, s}(x)\right\| \leq \exp ((t-s) \beta), \forall t \geq s \geq 0 .
$$

Proof. For $i=1, \ldots, d$ and $\mathcal{Y}_{t, s}(x)=\left(\mathcal{Y}_{t, s}^{1}(x), \ldots, \mathcal{Y}_{t, s}^{d}(x)\right)$,

$$
\partial_{x_{i}} \mathcal{Y}_{t, s}^{j}(x)=\delta_{i, j}+\int_{s}^{t} \sum_{k=1}^{n} \partial_{x_{k}} b_{j}\left(\mathcal{Y}_{s, r}(x)\right) \partial_{i} \mathcal{Y}_{s, r}^{k}(x) \mathrm{d} r
$$

After having considered formally this equation, it is then possible to show that $\nabla \mathcal{Y}_{t, s}(x)$ is really the derivative of $\mathcal{Y}_{t, s}(x)$.

By identifying $\nabla \mathcal{Y}_{t, s}(x)$ with a matrix $\left(\partial_{x_{i}} Y_{t, s}^{j}(x)\right)_{i, j=1, \ldots, d}$,

$$
\left\|\nabla \mathcal{Y}_{t, s}(x)\right\| \leq 1+\int_{s}^{t} \beta\left\|\nabla \mathcal{Y}_{r, s}(x)\right\| \mathrm{d} r
$$

so that (11) for $\nabla \mathcal{Y}_{t, s}(x)$ follows from the Grönwall lemma. Similarly,

$$
\partial_{x_{i}} \mathcal{X}_{t, s}^{j}(x)=\delta_{i, j}+\int_{s}^{t} \sum_{k=1}^{n} \partial_{x_{k}} b_{j}\left(\mathcal{X}_{r, s}(x)\right) \partial_{i} \mathcal{X}_{r, s}^{k}(x) \mathrm{d} r
$$

so that a control similar to the one on $\nabla \mathcal{Y}_{t}$ holds for $\nabla \mathcal{X}_{t, s}$.

The control over the derivative of the flow is then transferred as a control on the semi-groups.

Corollary 1. For any $t \geq s \geq 0$ and $g \in \mathrm{C}_{\mathrm{b}}^{1}\left(\mathbb{R}^{d}, \mathbb{R}\right)$,

$$
\left\|\nabla X_{s, t} g\right\|_{\infty} \leq \exp (\beta(t-s))\|\nabla g\|_{\infty} .
$$

Proof. For any $0 \leq s \leq t$, the chain rule implies that

$$
\nabla X_{s, t} g(x)=\nabla \mathbb{E}\left[g\left(\mathcal{X}_{t, s}(x)\right)\right]=\mathbb{E}\left[\nabla \mathcal{X}_{t, s}(x) \nabla g\left(\mathcal{X}_{t, s}(x)\right)\right]
$$

The result stems from 11 . 


\subsection{The heat semi-group}

Being associated to the heat equation, we call $\left(P_{s, t}\right)_{0 \leq s \leq t}$ the heat semi-group (see App. B.1.

Lemma 2. For any $0 \leq s \leq t$,

$$
\begin{gathered}
\left\|P_{s, t} g\right\|_{\infty} \leq\|g\|_{\infty}, g \in \mathrm{C}_{\mathrm{b}}\left(\mathbb{R}^{d}, \mathbb{R}\right) \\
\text { and }\left\|\nabla P_{s, t} g\right\|_{\infty} \leq\|\nabla g\|_{\infty}, g \in \mathrm{C}_{\mathrm{b}}^{1}\left(\mathbb{R}^{d}, \mathbb{R}\right) .
\end{gathered}
$$

In addition, for $g \in \mathrm{C}_{\mathrm{b}}\left(\mathbb{R}^{d}, \mathbb{R}\right)$ and $i=1, \ldots, d$,

$$
\partial_{x_{i}} P_{s, t} g(x)=\partial_{x_{i}} \mathbb{E}\left[g\left(\mathcal{B}_{t, s}(x)\right)\right]=\mathbb{E}\left[\frac{\mathrm{B}_{t}^{i}-\mathrm{B}_{s}^{i}}{t-s} g\left(\mathcal{B}_{t, s}(x)\right)\right] .
$$

For $\mu \in \mathbb{R}, \sigma>0$, let us denote by $\mathcal{N}(\mu, \sigma)$ the Gaussian distribution of mean $\nu$ and variance $\sigma$.

Proof. Inequalities (12) are immediate. For (13), since $x+\mathrm{B}_{t}-\mathrm{B}_{s} \sim \mathcal{N}(x, t-s)$,

$$
P_{s, t} g(x)=\int_{\mathbb{R}^{d}} \frac{1}{(2 \pi(t-s))^{d / 2}} \exp \left(\frac{-|y-x|^{2}}{2(t-s)}\right) g(x+y) \mathrm{d} y .
$$

Since

$$
\partial_{x_{i}} \exp \left(\frac{-|y-x|^{2}}{2(t-s)}\right)=\frac{\left(y_{i}-x_{i}\right)}{t-s} \exp \left(\frac{-|y-x|^{2}}{2(t-s)}\right)
$$

we obtain the integration by part formula (13).

Remark 1. An immediate consequence of $(13)$ is that

$$
\left\|\nabla P_{s, t} g\right\|_{\infty} \leq \frac{\|g\|_{\infty}}{\sqrt{t-s}}, \forall 0<s<t, \forall g \in \mathrm{C}_{\mathrm{b}}\left(\mathbb{R}^{d}, \mathbb{R}\right)
$$

\subsection{The transport semi-group}

The semi-group $\left(Q_{s, t}\right)_{0 \leq s \leq t}$ is associated to a transport equation (see App. B.2).

Lemma 3. For any $0 \leq s \leq t$,

$$
\left\|Q_{s, t} g\right\|_{\infty} \leq\|g\|_{\infty} \text { for } g \in \mathrm{C}_{\mathrm{b}}\left(\mathbb{R}^{d}, \mathbb{R}\right) .
$$

In addition,

$$
\left\|\nabla Q_{s, t} g\right\|_{\infty} \leq\|\nabla g\|_{\infty} \exp ((t-s) \beta), \forall t \geq 0 \text { for } g \in \mathrm{C}_{\mathrm{b}}^{1}\left(\mathbb{R}^{d}, \mathbb{R}\right) .
$$

Proof. Inequality (16) is immediate and (17) follows from Proposition 2. 
For $g \in \mathrm{C}_{\mathrm{b}}^{1}\left(\mathbb{R}^{d}, \mathbb{R}\right)$, the Newton formula implies that

$$
\begin{aligned}
g\left(\mathcal{Y}_{t, r}(x)\right)=g(x)+\int_{r}^{t} b\left(\mathcal{Y}_{s, r}(x)\right) & \nabla g\left(\mathcal{Y}_{s, r}(x)\right) \mathrm{d} s \\
& =g(x)+(t-r) b(x) \nabla g(x)+R(r, t, g, x)
\end{aligned}
$$

with a remainder term

$$
R(r, t, g, x)=\int_{r}^{t}\left(b\left(\mathcal{Y}_{s, r}(x)\right) \nabla g\left(\mathcal{Y}_{s, r}(x)\right)-b(x) \nabla g(x)\right) \mathrm{d} s .
$$

\section{The Euler scheme}

We give a first convergence result which is related to the Euler scheme.

Let us set $\mathcal{E}_{t, s}(x)=x+\mathrm{B}_{t}-\mathrm{B}_{s}+b(x)(t-s)=\mathcal{B}_{t, s}(x)+b(x)(t-s)$. This is one step of the Euler scheme, in the sense that for $t_{i}=i T / n, i=0, \ldots, n$,

$$
\xi_{i}(x)=\mathcal{E}_{t_{i}, t_{i-1}} \circ \cdots \circ \mathcal{E}_{t_{1}, 0}(x)
$$

and the $\xi_{i}(x)$ 's are easily recursively computed by

$$
\xi_{0}(x)=x \text { and } \xi_{i+1}(x)=\mathcal{E}_{t_{i+1}, t_{i}}\left(\xi_{i}(x)\right)=\xi_{i}+\mathrm{B}_{t_{i+1}}-\mathrm{B}_{t_{i}}+b\left(\xi_{i}(x)\right) \frac{T}{n}
$$

for $i=0, \ldots, n-1$.

The Euler scheme provides a simple way to approximate the flow $\mathcal{X}_{T, 0}(x)$ as

$$
\mathcal{E}_{T,(n-1) T / n} \circ \cdots \circ \mathcal{E}_{T / n, 0}(x) \underset{n \rightarrow \infty}{\longrightarrow} \mathcal{X}_{T, 0}(x) \text { almost surely. }
$$

We give an insight of the proof in Appendix A

In Proposition 3 below, an immediate consequence of the next lemma, we provide a weak rate of convergence for the Euler scheme.

Lemma 4. For $g \in \mathrm{C}_{\mathrm{b}}^{1}\left(\mathbb{R}^{d}, \mathbb{R}\right)$,

$$
\left\|E_{s, t} g-X_{s, t} g\right\|_{\infty} \leq\|\nabla g\|_{\infty} K(t-s)^{3 / 2} .
$$

Proof. Actually,

$$
\mathcal{X}_{t, s}(x)-\mathcal{E}_{s, t}(x)=\int_{s}^{t}\left(b\left(\mathcal{X}_{r, s}(x)\right)-b(x)\right) \mathrm{d} r
$$

so that with $\beta=\|\nabla b\|_{\infty}$,

$$
\left|\mathcal{X}_{t, s}(x)-\mathcal{E}_{t, s}(x)\right| \leq \beta \int_{s}^{t}\left|\mathrm{~B}_{r}-\mathrm{B}_{s}-\int_{s}^{r} b\left(\mathcal{X}_{u, s}(x)\right) \mathrm{d} u\right| \mathrm{d} r .
$$

Then for $g \in \mathrm{C}^{1}\left(\mathbb{R}^{d} ; \mathbb{R}\right)$,

$$
\left\|E_{s, t} g-X_{s, t} g\right\|_{\infty} \leq\|\nabla g\|_{\infty} \beta \int_{s}^{t} \mathbb{E}\left[\left|\mathrm{B}_{r}-\mathrm{B}_{s}\right|\right] \mathrm{d} r+\|\nabla g\|_{\infty} \frac{(t-s)^{2}}{2} \beta\|b\|_{\infty} .
$$

But $\mathbb{E}\left[\left|\mathrm{B}_{r}-\mathrm{B}_{s}\right|\right] \leq \sqrt{r-s}$. Hence the result. 
For $g \in \mathrm{C}_{\mathrm{b}}^{1}\left(\mathbb{R}^{d}, \mathbb{R}\right)$, let us denote

$$
\|g\|_{\star}=\max \left\{\|g\|_{\infty},\|\nabla g\|_{\infty}\right\} .
$$

For a bounded linear operator $A: \mathrm{C}_{\mathrm{b}}^{1}\left(\mathbb{R}^{d}, \mathbb{R}\right) \rightarrow \mathrm{C}_{\mathrm{b}}^{1}\left(\mathbb{R}^{d}, \mathbb{R}\right)$, let us set

$$
\|A\|_{\infty \rightarrow \infty}=\sup _{g \in \mathrm{C}_{\mathrm{b}},\|g\|_{\infty}=1}\|A g\|_{\infty} \text { and }\|A\|_{\star}=\sup _{g \in \mathrm{C}_{\mathrm{b}}^{1},\|g\|_{\star}=1}\|A g\|_{\star} .
$$

Proposition 3 (Weak rate of convergence of the Euler scheme). For any $g \in$ $\mathrm{C}_{\mathrm{b}}^{1}\left(\mathbb{R}^{d}, \mathbb{R}\right)$,

$$
\left\|\left(\prod_{i=0}^{n-1} E_{i T / n,(i+1) T / n}\right) g-X_{0, T} g\right\|_{\infty} \leq\|g\|_{\star} \frac{C}{\sqrt{n}},
$$

for a constant $C$ that depends only on $\|b\|_{\star}$ and $T$.

Proof. Writing $D_{s, t}=E_{s, t}-X_{s, t}$ and using Lemma 1

$$
\begin{aligned}
& E_{0,1 / n} \cdots E_{(n-1) T / n, T} g-X_{0,1 / n} \cdots X_{(n-1) T / n, T} g \\
& =\sum_{j=0}^{n-1}\left(\prod_{i=j+1}^{n-1} E_{i T / n,(i+1) T / n}\right) D_{(j-1) T / n, j T / n}\left(\prod_{i=0}^{j-1} X_{i T / n,(i+1) T / n}\right) g .
\end{aligned}
$$

With Lemma 4 , the product property 10 of $\left(X_{s, t}\right)$ and Corollary 1 ,

$$
\left\|D_{(j-1) T / n, j T / n} X_{0,(j-1) T / n} g\right\|_{\infty} \leq\|\nabla g\|_{\infty} K \frac{T^{3 / 2}}{n^{3 / 2}} .
$$

In addition, since $\left\|E_{s, t}\right\|_{\infty \rightarrow \infty} \leq 1$ and $\left\|E_{r, s} E_{s, t}\right\|_{\infty \rightarrow \infty} \leq\left\|E_{r, s}\right\|_{\infty \rightarrow \infty}\left\|E_{s, t}\right\|_{\infty \rightarrow \infty}$, on get easily (22) from the above inequality.

Remark 2. The weak rate of convergence of the Euler scheme is generally established for smoother coefficients $\left(\right.$ e.g. $\left.b \in \mathrm{C}^{4}\left(\mathbb{R}^{d}, \mathbb{R}^{d}\right)\right)$ to achieve a rate $1[49]$. In [38, 39], it is shown that for $\alpha$-Hölder continuous coefficients with $\alpha<2$, the order of convergence is $\alpha / 2$. This approach excludes the integer values of $\alpha$, and the terminal condition is required to be $(2+\alpha)$-Hölder continuous. With our regularity condition on the drift, we complete this result for $\alpha=1$ when the diffusivity is constant. This result can also be recovered with the results in [29].

Remark 3. For a closely related approach with semi-groups, the article [5] provides us with some conditions in a more general context to exhibit the rate of convergence of Euler schemes. 


\section{An intermediary convergence result}

We prove that the products of the operators $V_{s, t}$ over finer and finer partitions of $[0, T]$ converges to the operator $X_{0, T}$. This result is crucial to study the convergence of the products of $Q_{s, t} P_{s, t}$ in the next section.

Proposition 4. For any $g \in \mathrm{C}_{\mathrm{b}}^{1}\left(\mathbb{R}^{d}, \mathbb{R}\right)$,

$$
\left\|\left(\prod_{i=0}^{n-1} V_{i T / n,(i+1) T / n}\right) g-X_{0, T} g\right\|_{\infty} \leq\|g\|_{\star} \frac{C}{\sqrt{n}},
$$

for a constant $C$ that depends only on $\|b\|_{\star}$ and $T$.

Proof. From the Newton formula,

$$
g\left(\mathcal{E}_{t, s}(x)\right)=g\left(\mathcal{B}_{t, s}(x)\right)+(t-s) \int_{0}^{1} b(x) \nabla g\left(\mathcal{B}_{t, s}(x)+\tau(t-s) b(x)\right) \mathrm{d} \tau,
$$

so that

$$
\begin{aligned}
& \mid E_{s, t} g(x)- V_{s, t} g(x)|\leq| \mathbb{E}\left[g\left(\mathcal{E}_{t, s}(x)\right]-\mathbb{E}\left[g\left(\mathcal{B}_{t, s}(x)\right)\right]-\mathbb{E}\left[(t-s) b(x) \nabla g\left(\mathcal{B}_{t, s}(x)\right)\right] \mid\right. \\
& \leq(t-s) \int_{0}^{1}\|b\|_{\infty}\left|\mathbb{E}\left[\nabla g\left(\mathcal{B}_{t, s}(x)+\tau(t-s) b(x)\right)-\nabla g\left(\mathcal{B}_{t, s}(x)\right)\right] \mathrm{d} \tau\right|
\end{aligned}
$$

Again with an integration by parts on the density of the normal distribution, for $G \sim \mathcal{N}(0,1)$

$$
\mathbb{E}[\nabla \phi(\mu+\sigma G)]=\frac{1}{\sqrt{2 \pi}} \int \exp \left(\frac{-|y|^{2}}{2}\right) \nabla \phi(\mu+\sigma y) \mathrm{d} y=\mathbb{E}\left[\frac{G}{\sigma} \phi(\mu+\sigma G)\right],
$$

from which we obtain for $\tau \in[0,1]$,

$$
\mathbb{E}\left[\nabla g\left(\mathcal{B}_{t, s}(x)+\tau(t-s) b(x)\right)\right]=\mathbb{E}\left[\frac{\mathrm{B}_{t}-\mathrm{B}_{s}}{t-s} g\left(\mathcal{B}_{t, x}(x)+\tau(t-s) b(x)\right] .\right.
$$

Thus,

$$
\left|E_{s, t} g(x)-V_{s, t} g(x)\right| \leq \frac{\|b\|_{\infty}^{2}}{2} \mathbb{E}\left[\left|\mathrm{B}_{t}-\mathrm{B}_{s}\right|\right]\|\nabla g\|_{\infty}(t-s) \leq C\|\nabla g\|_{\infty}(t-s)^{3 / 2} .
$$

With Lemma 4

$$
\left|X_{s, t} g(x)-V_{s, t} g(x)\right| \leq C^{\prime}(t-s)^{3 / 2}\|\nabla g\|_{\infty} .
$$

The result follows from the same argument as in the proof of Proposition 3. 


\section{The splitting procedure}

The composition $Q_{s, t} P_{s, t}$ corresponds to a splitting (or composition): first, we follow the flow generated $\mathcal{B}$ starting from $x$ and then the one generated by $\mathcal{Y}_{\cdot, s}$ starting at $\mathcal{B}_{t, s}(x)$. The convergence of the products of $Q$ and $P$ over finer and finer partitions is the spirit of the Trotter-Kato-Lie approach to construct the semi-group $X$ (see 12,17$])$.

The main point is that if $g$ is $\mathrm{C}_{\mathrm{b}}^{1}(\mathbb{R}, \mathbb{R})$, then

$$
Q_{s, t} g(x)=g\left(\mathcal{Y}_{t, s}(x)\right)=g(x)+\int_{s}^{t} b \nabla g\left(\mathcal{Y}_{r, s}(x)\right) \mathrm{d} r=g(x)+(t-s) b \nabla g(x)+\cdots .
$$

We use this Taylor development to obtain some control over the product $Q_{s, t} P_{s, t}$, together with an integration by parts on the Brownian density.

Proposition 5. For any $g \in \mathrm{C}_{\mathrm{b}}^{1}\left(\mathbb{R}^{d}, \mathbb{R}\right)$,

$$
\left\|\left(\prod_{i=0}^{n-1} Q_{i T / n,(i+1) T / n} P_{i T / n,(i+1) T / n}\right) g-X_{T, 0} g\right\|_{\infty} \leq\|g\|_{\star} \frac{C}{\sqrt{n}},
$$

for a constant $C$ that depends only on $\|b\|_{\star}$ and $T$.

Proof. With (12) and (17),

$$
\left\|\nabla\left(Q_{t} P_{t}\right) g\right\|_{\infty} \leq \exp \left(t\|\nabla b\|_{\infty}\right)\left\|\nabla P_{t} g\right\|_{\infty} \leq \exp (t \beta)\|g\|_{\infty} .
$$

From (18),

$$
Q_{s, t} P_{s, t} g(x)=P_{s, t} g(x)+(t-s) b(x) \nabla P_{s, t} g(x)+R\left(s, t, P_{s, t} g, x\right)
$$

where the remainder term $R(s, t, \cdot, x)$ is defined by (19). With (13),

$$
Q_{s, t} P_{s, t} g(x)=V_{t, s} g(x)+R\left(s, t, P_{s, t} g, x\right) .
$$

Since $b \in \mathrm{C}_{\mathrm{b}}^{1}\left(\mathbb{R}^{d}, \mathbb{R}\right)$,

$$
\left|\mathcal{Y}_{t, s}(x)-x\right|=\left|\int_{s}^{t} b\left(\mathcal{Y}_{r, s}(x)\right) \mathrm{d} r\right| \leq\|b\|_{\infty}(t-s) .
$$

Again from (13),

$$
\begin{aligned}
R(s, t & \left., P_{s, t} g, x\right) \\
\quad= & \left.\int_{s}^{t} \mathbb{E}\left[\frac{\mathrm{B}_{t}-\mathrm{B}_{s}}{t-s} \cdot\left(b\left(\mathcal{Y}_{r, s}(x)\right) g\left(\mathcal{Y}_{r, s}(x)+\mathrm{B}_{t}-\mathrm{B}_{s}\right)-b(x) g\left(\mathcal{B}_{t, s}(x)\right)\right)\right) \mathrm{d} r\right]
\end{aligned}
$$


so that with $(23)$,

$$
\begin{aligned}
\left\|R\left(s, t, P_{s, t} g, \cdot\right)\right\|_{\infty} \leq \mathbb{E}\left[\frac{\left|\mathrm{B}_{t}-\mathrm{B}_{s}\right|}{t-s}\right] \int_{s}^{t}\left(\|\nabla b\|_{\infty}\|g\|_{\infty}+\|b\|_{\infty}\|\nabla g\|_{\infty}\right) r \mathrm{~d} r & \leq 2\|b\|_{\star}\|g\|_{\star}(t-s)^{3 / 2}
\end{aligned}
$$

This proves that

$$
\left\|\left(\prod_{i=0}^{n-1} Q_{i T / n,(i+1) T / n} P_{i T / n,(i+1) T / n}\right) g-\left(\prod_{i=0}^{n-1} V_{i T / n,(i+1) T / n}\right) g\right\|_{\infty} \leq\|g\|_{\star} \frac{C}{\sqrt{n}},
$$

and the result follows from Proposition 4.

\section{The weights and their limits: the Girsanov theorem}

The expression of the weight will be obtained by two ways: The first one involves the expression of the Euler scheme. For this, we need however a change of measure which is easily deduced from the explicit expression of the Gaussian density. The second one involves the expressions of $V$ in the splitting procedure. With the discrete time approximation, both expressions leads to different expressions for the weights, whose limit is however the same. The expression obtained by the Euler scheme is a discretization of the stochastic integral in the exponential weight given by (5). The ones obtained using the splitting is a discretization of the SDE (6) the exponential weights solve.

We have now all the elements to prove the Girsanov theorem with our approach.

Theorem 3 (Girsanov, simplified form). There exists a stochastic process $\left(\mathrm{Z}_{t}(x)\right)_{t \in[0, T]}$ given by

$$
\begin{aligned}
\mathrm{Z}_{t}(x) & =\exp \left(\int_{0}^{t} b\left(x+\mathrm{B}_{s}\right) \mathrm{dB}_{s}-\frac{1}{2} \int_{0}^{t}\left|b\left(x+\mathrm{B}_{s}\right)\right|^{2} \mathrm{~d} s\right) \\
\text { or } \mathrm{Z}_{t}(x) & =1+\int_{0}^{t} \mathrm{Z}_{s}(x) b\left(x+\mathrm{B}_{s}\right) \mathrm{dB}_{s}
\end{aligned}
$$

such that

$$
\mathbb{E}\left[g\left(\mathcal{X}_{T, 0}(x)\right)\right]=\mathbb{E}\left[\mathbf{Z}_{T}(x) g\left(\mathcal{B}_{T, 0}(x)\right)\right]
$$

for any bounded, measurable function $\mathrm{g}$.

The weight $\left(Z_{t}(x)\right)_{t \geq 0}$ will be identified as a limit, and stochastic analysis is needed for this. For this, we will rewrite the probabilistic representation of the operators given by both the Euler scheme and the splitting scheme as involving some exponential term, and then combine them. The expression (24) is related to the representation provided by the Euler scheme while (25) stems from the splitting scheme.

The proof of this theorem is then obtained by combining Propositions 7 and 8 below with Remark 5 . 


\subsection{Exponential representation of the one-step Euler scheme}

The following exponential representation is a direct consequence of the expression of the density of the Gaussian distribution.

Lemma 5. For any $x \in \mathbb{R}^{d}$ and $0 \leq s \leq t$,

$$
\mathbb{E}\left[g\left(\mathcal{E}_{t, s}(x)\right)\right]=E_{t, s} g(x)=\mathbb{E}\left[\mathbf{E}_{s, t}(x) g\left(\mathcal{B}_{t, s}(x)\right)\right]
$$

with

$$
\mathbf{E}_{s, t}(x)=\exp \left(b(x) \cdot\left(\mathrm{B}_{t}-\mathrm{B}_{s}\right)-\frac{(t-s)}{2}|b(x)|^{2}\right) .
$$

Proof. When $G \sim \mathcal{N}\left(0, \sigma^{2} \operatorname{Id}_{d \times d}\right)$ and $H \sim \mathcal{N}\left(\mu, \sigma^{2} \operatorname{Id}_{d \times d}\right)$, then for any measurable, bounded function $\phi: \mathbb{R}^{d} \rightarrow \mathbb{R}$,

$$
\begin{gathered}
\mathbb{E}[\phi(\mu+G)]=\mathbb{E}[\phi(H)]=\int \phi(x) \frac{\exp \left(\frac{-|x-\mu|^{2}}{2 \sigma^{2}}\right)}{\left(2 \pi \sigma^{2}\right)^{d / 2}} \mathrm{~d} x \\
=\int \phi(x) \exp \left(\frac{x \cdot \mu}{\sigma^{2}}-\frac{|\mu|^{2}}{2 \sigma^{2}}\right) \frac{\exp \left(\frac{-|x|^{2}}{2 \sigma^{2}}\right)}{\left(2 \pi \sigma^{2}\right)^{d / 2}} \mathrm{~d} x=\mathbb{E}[\Psi(G, \mu) \phi(G)] \\
\text { with } \Psi(G, \mu)=\exp \left(\frac{G \cdot \mu}{\sigma^{2}}-\frac{|\mu|^{2}}{2 \sigma^{2}}\right) .
\end{gathered}
$$

With

$$
\begin{aligned}
& \phi(y)=g(x+y), G=\mathrm{B}_{t}-\mathrm{B}_{s} \sim \mathcal{N}\left(0,(t-s) \mathrm{Id}_{d \times d}\right) \\
& \text { and } H=(t-s) b(x)+\mathrm{B}_{t}-\mathrm{B}_{s} \sim \mathcal{N}\left((t-s) b(x),(t-s) \operatorname{Id}_{d \times d}\right),
\end{aligned}
$$

this yields the result.

\subsection{The weights}

Let us set for $x \in \mathbb{R}^{d}$ and $0 \leq r \leq t$,

$$
\mathbf{V}_{r, t}(x)=1+b(x)\left(\mathrm{B}_{t}-\mathrm{B}_{r}\right) \text { so that } V_{r, t} g(x)=\mathbb{E}\left[\mathbf{V}_{r, t}(x) g\left(\mathcal{B}_{t, r}(x)\right)\right] \text {. }
$$

Proposition 6. For any $x \in \mathbb{R}^{d}, T>0$ and $n \in \mathbb{N}$,

$$
\begin{gathered}
\prod_{i=0}^{n-1} E_{i T / n,(i+1) T / n} g(x)=\mathbb{E}\left[\mathrm{Z}_{n, n}^{E}(x) g\left(\mathcal{B}_{t, 0}(x)\right)\right] \\
\text { and } \prod_{i=0}^{n-1} V_{i T / n,(i+1) T / n} g(x)=\mathbb{E}\left[\mathrm{Z}_{n, n}^{V}(x) g\left(\mathcal{B}_{t, 0}(x)\right)\right] \\
\text { with } \mathbf{Z}_{k, n}^{E}(x)=\prod_{i=0}^{k-1} \mathbf{E}_{i T / n,(i+1) T / n}\left(\mathcal{B}_{i T / n, 0}(x)\right) \\
\text { and } \mathbf{Z}_{k, n}^{V}(x)=\prod_{i=0}^{k-1} \mathbf{V}_{i T / n,(i+1) T / n}\left(\mathcal{B}_{i T / n, 0}(x)\right) .
\end{gathered}
$$


Proof. Let us work on $E_{t, s}$, the proof being similar for $V_{t, s}$.

Set $F(x)=\mathbb{E}\left[g\left(\mathcal{E}_{t, s}(x)\right)\right]$ so that

$$
\begin{aligned}
& E_{r, s} E_{s, t} g(x)=\mathbb{E}\left[E_{s, t} g\left(\mathcal{E}_{s, r}(x)\right)\right]=\mathbb{E}\left[F\left(\mathcal{E}_{s, r}(x)\right)\right]=\mathbb{E}\left[\mathbf{E}_{r, s}(x) F\left(\mathcal{B}_{s, r}(x)\right)\right] \\
& \quad=\mathbb{E}\left[\mathbf{E}_{r, s}(x) \mathbf{E}_{s, t}\left(\mathcal{B}_{s, r}(x)\right) g\left(\mathcal{B}_{t, s}\left(\mathcal{B}_{s, r}(x)\right)\right)\right]=\mathbb{E}\left[\mathbf{E}_{r, s}(x) \mathbf{E}_{s, t}\left(\mathcal{B}_{s, r}(x)\right) g\left(\mathcal{B}_{t, r}(x)\right)\right]
\end{aligned}
$$

By iterating this computation, this leads to 26 .

\subsection{Uniform integrability of the weights}

For $n \geq 1$ and $T>0$ fixed, let us denote by $\Delta_{i}^{n} \mathrm{~B}=\Delta_{i T / n,(i+1) T / n}^{n} \mathrm{~B}$, the increments of the Brownian motion. For $i=1, \ldots, n$, set $\mathcal{F}_{i}^{n}=\sigma\left(\Delta_{0}^{n} \mathrm{~B}, \ldots, \Delta_{i-1}^{n} \mathrm{~B}\right)$, so that $\left(\mathcal{F}_{i}^{n}\right)_{i \geq 0}$ is the filtration generated by the increments of the Brownian motion.

We fix a starting point $x \in \mathbb{R}^{d}$.

Lemma 6. For each $n$, both $\left(\mathrm{Z}_{k, n}^{E}(x)\right)_{k \geq 0}$ and $\left(\mathrm{Z}_{k, n}^{V}(x)\right)_{n \geq 0}$ are discrete martingales with respect to $\left(\mathcal{F}_{i}^{n}\right)_{i=1, \ldots, n}$. Besides $\mathbb{E}\left[\bar{Z}_{k, n}^{E}(x)\right]=\mathbb{E}\left[\mathrm{Z}_{k, n}^{V}(x)\right]=1$.

Proof. The proof is immediate using the independence of the increments of the Brownian motion.

Remark 4. Although $Z_{n, n}^{E}(x)$ and $Z_{n, n}^{V}(x)$ are close, they are certainly not equal as $Z_{n, n}^{E}(x)$ remains positive while $Z_{n, n}^{V}(x)$ is negative with a positive probability.

To be able to pass to the limit, we prove that the family weights are uniformly integrable martingales.

Lemma 7. Both $\left(\mathrm{Z}_{k, n}^{E}(x)\right)_{n \geq 1}$ and $\left(\mathrm{Z}_{k, n}^{V}(x)\right)_{n \geq 1}$ are uniformly integrable.

Remark 5. Provided that $Z_{n, n}^{E}(x)$ and $Z_{n, n}^{V}(x)$ converge in distribution to some random variable $Z_{T}$, the uniform integrability of these random variables is sufficient to assert that

$$
\lim _{n \rightarrow \infty} \mathbb{E}\left[\mathbf{Z}_{n, n}^{E}(x) g\left(\mathcal{B}_{T, 0}(x)\right)\right]=\lim _{n \rightarrow \infty} \mathbb{E}\left[\mathbf{Z}_{n, n}^{V}(x) g\left(\mathcal{B}_{T, 0}(x)\right)\right]=\mathbb{E}\left[\mathbf{Z}_{T}(x) g\left(\mathcal{B}_{T, 0}(x)\right)\right] .
$$

Proof. Using the independence of the increment and the property of the Laplace transform of the Gaussian distribution,

$$
\mathbb{E}\left[\left(\exp \left(b\left(x+\mathrm{B}_{i T / n}\right) \Delta_{i}^{n} \mathrm{~B}-\frac{T}{2 n}\left|b\left(x+\mathrm{B}_{i T / n}\right)\right|^{2}\right)\right)^{2} \mid \mathcal{F}_{i}^{n}\right] \leq \exp \left(\|b\|_{\infty} \frac{T}{n}\right),
$$

so that, by computing iteratively the conditional expectations,

$$
\sup _{\substack{k=1, \ldots, n \\ n \geq 1}} \mathbb{E}\left[\left(Z_{k, n}^{E}(x)\right)^{2}\right] \leq \exp \left(\|b\|_{\infty} T\right) .
$$


Similarly,

$$
\mathbb{E}\left[\left(1+b\left(x+\mathrm{B}_{i T / n}\right) \Delta_{i}^{n} \mathrm{~B}\right)^{2} \mid \mathcal{F}_{i}^{n}\right] \leq 1+\frac{T}{n}\|b\|_{\infty} \leq \exp \left(\|b\|_{\infty} \frac{T}{n}\right),
$$

so that

$$
\sup _{\substack{k=1, \ldots, n \\ n \geq 1}} \mathbb{E}\left[\left(Z_{k, n}^{V}(x)\right)^{2}\right] \leq \exp \left(\|b\|_{\infty} T\right) .
$$

This is sufficient to prove and uniform integrability.

\subsection{Identification of the limit of the weights: stochastic calculus at last}

At this stage, we need some stochastic calculus in order to identify the limit of $\mathrm{Z}_{k, n}^{E}(x)$ and $\mathrm{Z}_{k, n}^{V}(x)$.

Let us rewrite $\mathbf{Z}_{k, n}^{E}(x)$ as the iterative family by setting $\mathbf{Z}_{0, n}^{E}(x)=1$ and

$$
\begin{aligned}
Z_{k+1, n}^{E}(x) & =\mathrm{Z}_{k, n}^{E}(x) \exp \left(b\left(x+\mathrm{B}_{t_{k}^{n}}\right) \Delta_{k}^{n} \mathrm{~B}-\frac{T}{2 n}\left|b\left(x+\mathrm{B}_{t_{k}^{n}}\right)\right|^{2}\right) \\
& =\exp \left(\sum_{i=0}^{k} b\left(x+\mathrm{B}_{t_{i}^{n}}\right) \Delta_{i}^{n} \mathrm{~B}-\frac{T}{2 n} \sum_{i=0}^{k}\left|b\left(x+\mathrm{B}_{t_{i}^{n}}\right)\right|^{2}\right)
\end{aligned}
$$

for $k=0, \ldots, n-1$.

The next result is an immediate consequence of the definition of a stochastic integral. We recover the traditional expression for the Girsanov weight.

Proposition 7. When $n \rightarrow \infty$ and $k_{n} \rightarrow \infty$ with $k_{n} / n \underset{n \rightarrow \infty}{\longrightarrow}$ for some $t \in[0, T]$, $\mathrm{Z}_{k_{n}, n}^{E}(x)$ converges in probability to $\mathrm{Z}_{t}(x)$ given by (24).

An application of the Itô formula yields the well known fact that $Z_{t}$ given by (24) is solution to the Stochastic Differential Equation (25).

On the other hand, writing $\mathbf{Z}_{k, n}^{V}(x)$ as an iterative family yields that

$$
Z_{k+1, n}^{V}(x)=Z_{k, n}^{V}\left(1+b\left(x+\mathrm{B}_{t_{k}^{n}}\right) \Delta_{k}^{n} \mathrm{~B}\right) \text { for } k=0, \ldots, n-1
$$

with $\mathrm{Z}_{0, n}^{V}=1$. This could immediately be rewritten as the discrete analogue of 25 by

$$
Z_{k+1, n}^{V}(x)=1+\sum_{i=0}^{k} \mathrm{Z}_{i, n}^{V}(x) b\left(x+\mathrm{B}_{t_{i}^{n}}\right) \Delta_{i}^{n} \mathrm{~B} \text { for } k=0, \ldots, n-1 .
$$

The convergence of $Z_{k, n}^{V}(x)$ is less immediate than the one of $Z_{k, n}^{E}(x)$. Hopefully, it can be dealt with the results of D. Duffie and P. Protter 16 (see also 30, Example 8.7, p. 33]).

Proposition 8. When $n \rightarrow \infty$ and $k_{n} \rightarrow \infty$ with $k_{n} / n \underset{n \rightarrow \infty}{\longrightarrow}$ t for some $t \in[0, T]$, $\mathrm{Z}_{k_{n}, n}^{V}(x)$ converges in distribution to $\mathrm{Z}_{t}(x)$ given by (25) or equivalently (24). 


\section{The infinitesimal generator of the semi-group $\left(X_{t}\right)_{t \geq 0}$}

We compute now the infinitesimal generator of $\left(X_{t}\right)_{t \geq 0}$, still using only functional analysis. For the details, we refer for example to the book [12, 17].

The semi-groups $\left(X_{s, t}\right)_{0 \leq s \leq t},\left(P_{s, t}\right)_{0 \leq s \leq t}$ and $\left(Q_{s, t}\right)_{0 \leq s \leq t}$ are indeed homogeneous in time, as actually $X_{s, t}=X_{0, t-s}, P_{s, t}=P_{0, t-s}$ and $Q_{s, t}=Q_{0, t-s}$. We now set $X_{t}=X_{0, t-s}, P_{t}=P_{0, t-s}$ and $Q_{t}=Q_{0, t-s}$.

Definition 1 (Strongly continuous semi-group). Let B be a Banach space with a norm $\|\cdot\|$. A semi-group $\left(F_{t}\right)_{t \geq 0}$ on $\mathrm{B}$ is said to be strongly continuous if for any $f \in \mathrm{B}, T_{f} f$ is continuous.

Definition 2 (Infinitesimal generator). The infinitesimal generator of a semigroup $\left(F_{t}\right)_{t \geq 0}$ on $\mathrm{B}$ is a linear operator $\mathfrak{F}: \operatorname{Dom}(\mathfrak{F}) \subset \mathrm{B} \rightarrow \mathrm{B}$ such that

$$
\operatorname{Dom}(\mathfrak{F})=\left\{\begin{array}{l|l|l}
f \in \mathrm{B} & \left.\lim _{h \rightarrow 0} \frac{F_{h} f-f}{h} \text { exists }\right\}
\end{array}\right.
$$

and $\mathfrak{F} f=\lim _{h \rightarrow 0}\left(F_{h} f-f\right) / h$.

When $(\mathfrak{F}, \operatorname{Dom}(\mathfrak{F}))$ is closed for the graph norm $\|\cdot\|+\|\mathfrak{F} \cdot\|$ and densely defined in $\mathrm{B}$, then it determines the semi-group uniquely [17, Proposition 1.4, p. 51].

We consider that the underlying Banach space is $\mathrm{B}=\mathrm{C}_{\mathrm{z}}\left(\mathbb{R}^{d}, \mathbb{R}\right)$, the space of continuous, bounded functions that vanish at infinity, and $\|\cdot\|=\|\cdot\|_{\infty}$. We denote by $\mathrm{C}_{\mathrm{c}}^{k}\left(\mathbb{R}^{d}, \mathbb{R}\right) \subset \mathrm{C}_{\mathrm{z}}\left(\mathbb{R}^{d}, \mathbb{R}\right)$ the space of functions of class $\mathrm{C}^{k}\left(\mathbb{R}^{d}, \mathbb{R}\right)$ with compact support.

On $\mathrm{B}$, we consider the heat operator $\mathfrak{L}=\frac{1}{2} \triangle$, whose domain is the closure of $\mathrm{C}_{\mathrm{c}}^{2}\left(\mathbb{R}^{d}, \mathbb{R}\right)$ for the graph norm. This is the infinitesimal generator of $\left(P_{t}\right)_{t \geq 0}$ (see App. B.1).

We also consider $\mathfrak{B}=b \nabla$ whose domain is the closure of $\mathrm{C}_{\mathrm{c}}^{1}\left(\mathbb{R}^{d}, \mathbb{R}\right)$ for the graph norm. The operator $(\mathfrak{B}, \operatorname{Dom}(\mathfrak{B}))$ is the infinitesimal generator of $\left(Q_{t}\right)_{t \geq 0}$ (See App. B.2).

The next result is an "almost" direct consequence of the Trotter-Kato-Lie formula.

Proposition 9. The infinitesimal generator of the semi-group $\left(X_{t}\right)_{t \geq 0}$ is $\mathfrak{A}=$ $\mathfrak{L}+\mathfrak{B}$ with $\operatorname{Dom}(\mathfrak{A})=\operatorname{Dom}(\mathfrak{L})$.

Proof. To construct the semi-group $\left(X_{t}\right)_{t \geq 0}$, we apply Corollary 5.8 in [17, p. 227], which follows from the Chernoff product formula [17, Theorem 5.2, p. 220].

We show first that $\operatorname{Dom}(\mathfrak{L}) \subset \operatorname{Dom}(\mathfrak{B})$.

For any $\lambda>0,(\lambda-\mathfrak{L})$ is invertible on $\mathrm{B}$ and $\operatorname{Dom}(\mathfrak{L})=(\lambda-\mathfrak{L})^{-1}(\mathrm{~B})$. Moreover,

$$
(\lambda-\mathfrak{L})^{-1} f(x)=\int_{0}^{+\infty} e^{-\lambda t} P_{t} f(x) \mathrm{d} t .
$$


Using the control 15$)$, for any $f \in \mathrm{B}$,

$$
\left\|\nabla(\lambda-\mathfrak{L})^{-1} f(x)\right\|_{\infty} \leq C(\lambda)\|f\|_{\infty}
$$

with $C(\lambda)=\int_{0}^{+\infty} t^{-1 / 2} e^{-\lambda t} \mathrm{~d} t$. Thus,

$$
\left\|\mathfrak{B}(\lambda-\mathfrak{L})^{-1} f\right\|_{\infty} \leq\|b\|_{\infty} C(\lambda)\|f\|_{\infty} .
$$

It follows that $\mathfrak{B}$ is well defined for any $\operatorname{Dom}(\mathfrak{L})$ since for any $g \in \operatorname{Dom}(\mathfrak{L})$, there exists $f \in \mathrm{B}$ such that $g=(\lambda-\mathfrak{L})^{-1} f$.

Both $\left(Q_{t}\right)_{t \geq 0}$ and $\left(P_{t}\right)_{t \geq 0}$ are contraction semi-groups 8 , since $\left\|P_{t} g\right\|_{\infty} \leq\|g\|_{\infty}$ and $\left\|Q_{t} g\right\|_{\infty} \leq\|g\|_{\infty}$ for any $g \in \mathrm{B}=\mathrm{C}_{\mathrm{z}}\left(\mathbb{R}^{d}, \mathbb{R}\right)$. Thus, for any $t \geq 0, P_{t} Q_{t}$ is a bounded operator on B with norm 1.

It remains to show that $(\lambda-\mathfrak{L}-\mathfrak{B})(\operatorname{Dom}(\mathfrak{L}))$ is dense in $\mathrm{B}=\mathrm{C}_{\mathrm{z}}\left(\mathbb{R}^{d}, \mathbb{R}\right)$ for some $\lambda>0$. We actually show that $(\lambda-\mathfrak{L}-\mathfrak{B})$ is one-to-one between $\operatorname{Dom}(\mathfrak{L})$ to B.

For this, we consider finding the pairs $(f, g) \in \operatorname{Dom}(\mathfrak{L}) \times$ B such that

$$
(\lambda-\mathfrak{L}-\mathfrak{B}) f=g .
$$

We rewrite (29) as

$$
f-(\lambda-\mathfrak{L})^{-1} \mathfrak{B} f=(\lambda-\mathfrak{L})^{-1} g,
$$

so that $f$ is sought as

$$
f=\mathfrak{K} g=\lim _{n \rightarrow \infty} \mathfrak{K}_{n} g \text { with } \mathfrak{K}_{n} g=\sum_{k=0}^{n}\left((\lambda-\mathfrak{L})^{-1} \mathfrak{B}\right)^{k}(\lambda-\mathfrak{L})^{-1} g .
$$

For any $k \geq 1$,

$$
\left((\lambda-\mathfrak{L})^{-1} \mathfrak{B}\right)^{k}(\lambda-\mathfrak{L})^{-1}=(\lambda-\mathfrak{L})^{-1}\left(\mathfrak{B}(\lambda-\mathfrak{L})^{-1}\right)^{k} .
$$

Inequality (8) proves that $\mathfrak{B}(\lambda-\mathfrak{L})^{-1}$ is a bounded operator on $\mathrm{B}$ with constant $\|b\|_{\infty} C(\lambda)$.

When $\lambda \rightarrow \infty, C(\lambda)$ decreases to 0 . We choose $\lambda$ large enough so that $\|b\|_{\infty} C(\lambda)<$ 1. An immediate consequence of $(27)$ is that $(\lambda-\mathfrak{L})^{-1}$ is bounded by $1 / \lambda$. Thus, for any $k \geq 1$,

$$
\left\|\left((\lambda-\mathfrak{L})^{-1} \mathfrak{B}\right)^{k}(\lambda-\mathfrak{L})^{-1} g\right\|_{\infty} \leq \frac{1}{\lambda}\left(\|b\|_{\infty} C(\lambda)\right)^{k}\|g\|_{\infty} .
$$

${ }^{8}$ These semi-groups satisfies the far more finer properties of being Feller, as for $\left(X_{t}\right)_{t \geq 0}$, but we do not use it here. 
This means that the series $\mathfrak{K} g$ defined in 30 converges in B. Moreover, it is easily checked that

$$
(\lambda-\mathfrak{L}-\mathfrak{B}) \mathfrak{K}_{n}(\lambda-\mathfrak{L})^{-1} g=-\left(\mathfrak{B}(\lambda-\mathfrak{L})^{-1}\right)^{n+1} g+g .
$$

With (28), under the condition that $C(\lambda)\|b\|_{\infty}<1, f=\mathfrak{K} g$ solves $(\lambda-\mathfrak{L}-\mathfrak{B}) f=g$ in $\mathrm{B}$.

Since

$$
\mathfrak{B} \mathfrak{K}_{n} g=\sum_{k=0}^{n} \mathfrak{B}\left[(\lambda-\mathfrak{L})^{-1} \mathfrak{B}\right]^{k}(\lambda-\mathfrak{L})^{-1} g=\sum_{k=1}^{n+1}\left[\mathfrak{B}(\lambda-\mathfrak{L})^{-1}\right]^{k} g,
$$

it follows from (8) that when $C(\lambda)\|b\|_{\infty}<1,\left(\mathfrak{B} \mathfrak{K}_{n} g\right)_{n \geq 1}$ forms a Cauchy sequence. Since $(\mathfrak{B}, \operatorname{Dom}(\mathfrak{B}))$ is a closed operator, its limit is necessarily $\mathfrak{B} \mathfrak{K} g$. Thus,

$$
(\lambda-\mathfrak{L}) \mathfrak{K}_{n} g=g-\left(\mathfrak{B}(\lambda-\mathfrak{L})^{-1}\right)^{n+1} g+\mathfrak{B} \mathfrak{K}_{n} g .
$$

Clearly, $\mathfrak{K}_{n} g \in \operatorname{Dom}(\mathfrak{L})$. Since $(\mathfrak{L}, \operatorname{Dom}(\mathfrak{L}))$ is also a closed operator, we obtain that by passing to the limit, $\mathfrak{K} g \in \operatorname{Dom}(\mathfrak{L})$ and

$$
(\lambda-\mathfrak{L}) \mathfrak{K} g=g+\mathfrak{B} \mathfrak{K} g
$$

so that $\lambda-\mathfrak{L}-\mathfrak{B}$ is invertible from $\operatorname{Dom}(\mathfrak{L})$ to $\mathrm{B}$ with inverse $\mathfrak{K}$ which is bounded on B. This implies that $(\mathfrak{L}+\mathfrak{B})$ is itself a closed operator, with domain $\operatorname{Dom}(\mathfrak{L})$. The latter domain is dense in B.

The Chernoff product formula [17, Theorem 5.2, p. 220] then proves that the infinitesimal generator of $\left(X_{t}\right)_{t \geq 0}$ is $(\mathfrak{L}+\mathfrak{B}, \operatorname{Dom}(\mathfrak{L}+\mathfrak{B}))$.

\section{A case where Itô formula could be avoided (or Feynman, Kac, Girsanov and Doob meet together)}

Still to stuck to our rule to avoid stochastic calculus for the sake of play, we show that for special form of the drift, an already known probabilistic representation can be obtained by combining the previous results. The representation is the starting point of the so-called exact simulation method from A. Beskos and G.O. Roberts 7 for simulating a Brownian motion with drift by performing an acceptance/rejection scheme on paths from the Brownian bridge.

\subsection{The Feynman-Kac formula}

The Feynman-Kac formula provides a probabilistic representation to the solution to

$$
\left\{\begin{array}{l}
\partial_{t} u(t, x)=\frac{1}{2} \triangle u(t, x)+U(x) u(t, x) \\
u(0, x)=f(x)
\end{array}\right.
$$


Theorem 4 (M. Kac 27]). Let $U$ be bounded , Then the solution of (31) is solution to

$$
u(t, x)=\mathbb{E}\left[\exp \left(\int_{0}^{t} U\left(x+\mathrm{B}_{s}\right) \mathrm{d} s\right) f\left(x+\mathrm{B}_{t}\right)\right] .
$$

Proved first by M. Kac, it is also related to the Feynman path integral for solving the Schrödinger equation (See e.g. [40, 45]). This formula has many applications, in mathematical physics of course, but also in analysis. For example, it provides an effective way to compute Laplace transforms of functionals of the Brownian motion - the original goal of M. Kac 27] (for applications, see e.g. [25]) or to perform some change of measures 44 .

The Feynman-Kac formula could also be proved naturally through the TrotterKato-Lie formula (see e.g. [21, 48]).

With $\mathfrak{L}=\frac{1}{2} \triangle$ and $\mathfrak{U}$ be defined on the space of bounded functions by $\mathfrak{U} f(x)=$ $U(x) f(x)$, the semi-group $\left(U_{t}\right)_{t \geq 0}$ of $\mathfrak{U}$ is simply given by

$$
U_{t} f(x)=\exp (U(x) t) f(x), t \geq 0,
$$

a fact which is easily verified. Thus, by a computation similar to the one of Proposition 6, for any $t \geq 0$,

$$
\left(U_{t / n} P_{t / n}\right)^{n}=\mathbb{E}\left[\exp \left(\sum_{i=0}^{n-1} U\left(x+\mathrm{B}_{i t / n}\right) \frac{t}{n}\right) f\left(\mathrm{~B}_{t}\right)\right] \underset{n \rightarrow \infty}{\longrightarrow} u(t, x) \text { given by } 32 \text {. }
$$

On the other hand, the Trotter-Kato-Lie formula implies that

$\partial_{t} v(t, x)=(\mathfrak{L}+\mathfrak{U}) v(t, x)$ with $v(0, x)=f(x)$ when $v(t, x)=\lim _{n \rightarrow \infty}\left(U_{t / n} P_{t / n}\right)^{n} f(x)$.

\subsection{The infinitesimal generator of a semi-group under a $h$-transform}

Given a semi-group $\left(P_{t}\right)_{t>0}$ and a positive function $\phi$, one could naturally set

$$
P_{t}^{\phi} f=\frac{1}{\phi} P_{t}(\phi f), t \geq 0 .
$$

The rationale is that for any measurable, bounded function $f$,

$$
P_{t}^{\phi} P_{s}^{\phi} f=\frac{1}{\phi} P_{t}\left(\frac{\phi}{\phi} P_{s}(\phi f)\right)=\frac{1}{\phi} P_{t+s}(\phi f)=P_{t+s}^{\phi} f \text { for any } s, t \geq 0
$$

so that $\left(P_{t}^{\phi}\right)_{t \geq 0}$ is still a semi-group.

\footnotetext{
${ }^{9}$ This condition is stronger than the one given in the original article of M. Kac on that subject.
} 
Proposition 10. Let $\phi$ be a positive function of class $\mathrm{C}_{\mathrm{b}}^{2}\left(\mathbb{R}^{d}, \mathbb{R}^{d}\right)$. Consider the semi-group $\left(P_{t}\right)_{t \geq 0}$ generated by $\mathfrak{P}=\mathfrak{L}+\mathfrak{V}$. Then the infinitesimal generator $\mathfrak{P}^{\phi}$ of $\left(P_{t}^{\phi}\right)_{t \geq 0}$ is given by

$$
\mathfrak{P}^{\phi} f=\mathfrak{P} f+\frac{1}{\phi} \nabla \phi \cdot \nabla f+\frac{1}{2 \phi}(\triangle \phi) f .
$$

Since $\phi$ is positive, let us write $\Phi=\ln (\phi)$ so that for $f \in \mathrm{C}_{\mathrm{b}}^{2}\left(\mathbb{R}^{d}, \mathbb{R}^{d}\right)$,

$$
\mathfrak{P}^{\phi} f=\mathfrak{L} f+\mathfrak{V} f+\nabla \Phi \cdot \nabla f+\frac{1}{2}(\triangle \Phi) f+\frac{1}{2}(\nabla \Phi \cdot \nabla \Phi) f .
$$

Remark 6. If $\phi$ is a harmonic function, then $\mathfrak{P}^{\phi}$ is the infinitesimal generator of a diffusion process with drift $\nabla \phi / \phi$. This is the spirit of the h-transform or Doob's transform introduced by J.L. Doob [15] for solving problem in potential analysis. This implies a large class of processes with conditioning (for example, to construct a Brownian bridge where the value at a given time is fixed) could be obtained through a process with a drift (Actually, the same computations hold when $\phi$ is also time dependent).

\subsection{An alternative formulation for the Girsanov weights for some spe- cial form of the drift}

Given a bounded function $\phi$, it is tempting but hopeless to look for a function $\phi$ such that $\nabla \phi=\phi b$ and $\triangle \phi=0$. We are however free to choose the potential $U$.

Let $\left(S_{t}\right)_{t \geq 0}$ be the semi-group generated by $\mathfrak{P}=\mathfrak{L}+\mathfrak{U}$ with $U=-\frac{1}{2} \nabla \Phi \cdot \nabla \Phi+$ $\frac{1}{2} \triangle \Phi$ with $\Phi=\log \phi$. Hence,

$$
\begin{aligned}
& S_{t}^{\phi} f(x) \\
= & \frac{1}{e^{\Phi(x)}} \mathbb{E}_{x}\left[\exp \left(-\frac{1}{2} \int_{0}^{t} \nabla \Phi\left(\mathrm{B}_{s}\right) \cdot \nabla \Phi\left(\mathrm{B}_{s}\right) \mathrm{d} s+\int_{0}^{t} \frac{1}{2} \triangle \Phi\left(\mathrm{B}_{s}\right) \mathrm{d} s+\Phi\left(\mathrm{B}_{t}\right)\right) f\left(\mathrm{~B}_{t}\right)\right] .
\end{aligned}
$$

According to the above rules, the infinitesimal generator $\mathfrak{S}^{\phi}$ of $S_{t}^{\phi}$ is

$$
\mathfrak{S}^{\phi} f=\mathfrak{L} f+\nabla \Phi \cdot \nabla f .
$$

Proposition 11. Assume that $b \in \mathrm{C}^{1}\left(\mathbb{R}^{d}, \mathbb{R}^{d}\right)$ is a potential vector field, i.e., there exists $\Phi \in \mathrm{C}^{2}\left(\mathbb{R}^{d}, \mathbb{R}\right)$ such that $b=\nabla \Phi$. Then for a bounded, measurable function $f, \mathbb{E}\left[f\left(X_{t}\right)\right]=S_{t}^{\phi} f(x)$ where $S_{t}^{\phi}$ is given by (33).

Actually, (33) is not surprising. With the Itô's formula,

$$
\Phi\left(\mathrm{B}_{t}\right)-\Phi\left(\mathrm{B}_{0}\right)=\int_{0}^{t} \nabla \Phi\left(\mathrm{B}_{s}\right) \mathrm{dB}_{s}-\frac{1}{2} \int_{0}^{t} \triangle \Phi\left(\mathrm{B}_{s}\right) \mathrm{d} s,
$$

from which the classical representation of the exponential weight of the Girsanov theorem is easily obtained. 


\section{Complement: On the regularity of the drift and the difference between classical and stochastic analysis}

With our approach, the drift was assumed to be Lipschitz continuous. The main reason for this condition is that from the very construction, the flow associated to the ODE $\dot{\mathbf{X}}=b(\mathbf{X})$ needs to be defined to construct the semi-group $\left(Q_{t}\right)_{t>0}$.

If $b$ is not Lipschitz continuous, the equation $\dot{\mathrm{X}}=b(\mathrm{X})$ may have several solutions. A classical example is $b(x)=\sqrt{x}$.

The regularity of $b$ could be weakened to define a flow or to consider a particular solution to $\dot{\mathrm{X}}=b(\mathrm{X})$ but still a minimal regularity of $b$ should be enforced. For example, $b$ should belong to some Sobolev space [2, 13].

On the other hand, the situation changes when SDE are considered. Striking results from A. Zvonkin [53] and afterwards A. Veretennikov [52 prove the existence of a unique strong solution to the SDE

$$
\mathrm{dX}_{t}=\sigma\left(\mathbf{X}_{t}\right) \mathrm{dB}_{t}+b\left(\mathbf{X}_{t}\right) \mathrm{d} t
$$

whatever the regularity of $b$ provided that

$$
\mathrm{dX}_{t}=\sigma\left(\mathbf{X}_{t}\right) \mathrm{dB}_{t}, t \geq 0
$$

has a strong solution (of course, with suitable integrability conditions on $b$ ). The solution to (34) defines a flow of diffeomorphisms when $\sigma$ is a positive constant and $b$ is Hölder continuous.

Taking $\sigma(z)=\epsilon z$ for $\epsilon>0$ which is taken as small as possible proved the uniqueness of a solution to the SDE $\mathrm{dX}_{t}=\epsilon \mathrm{dB}_{t}+b\left(\mathrm{X}_{t}\right) \mathrm{d} t$ seen as a noisy perturbation of the ODE $\dot{X}=b(\mathrm{X})$ even when the latter ODE has no single solution. This fact is discussed among other references in [3, 19].

For the Girsanov theorem seen as a change of measure, the regularity of $b$ plays no role. One has only to ensure that the exponential super-martingale $Z_{t}=$ $\exp \left(\int_{0}^{t} b\left(\mathbf{X}_{s}\right) \mathrm{dB}_{s}+\frac{1}{2} \int_{0}^{t} b\left(\mathbf{X}_{s}\right) \mathrm{d} s\right)$ defining the weight is actually a martingale, and numerous conditions have been given on $b$ (See e.g. [20, 33]). For this, the drift appears only in an integrated form. An immediate application is the existence of a weak solution to the (34) when (35) has one, whatever the regularity of $b$ (of course, with the suitable integrability conditions on $b$, see e.g. $47 \mid)$.

We then see that a difference holds by considering (35) and $\dot{X}=b(\mathrm{X})$ separately through the Trotter-Kato-Lie, or by considering directly SDE of type (34), which draws a line of separation between stochastic and ordinary differential equations. This explains why our conditions on $b$ are stronger than the one required usually when invoking the Girsanov theorem. 


\section{A Almost sure convergence of the Euler scheme}

The convergence property 20 of the Euler scheme follows from the recursive inequality

$$
\begin{array}{r}
\left|\xi_{i+1}(x)-\mathcal{X}_{t_{i+1}, 0}(x)\right| \leq\left|\xi_{i}(x)-\mathcal{X}_{t_{i}, 0}(x)\right|+\rho_{i}+\frac{T}{n}\left|b\left(\xi_{i}(x)\right)-b\left(\mathcal{X}_{t_{i}, 0}(x)\right)\right| \\
\leq \exp \left(T\|\nabla b\|_{\infty}\right) \sum_{j=1}^{i} \rho_{j}
\end{array}
$$

with

$$
\rho_{i}=\int_{t_{i}}^{t_{i+1}}\left|b\left(\mathcal{X}_{r, 0}(x)\right)-b\left(\mathcal{X}_{t_{i}, 0}(x)\right)\right| \mathrm{d} r \leq \frac{T}{n}\|\nabla b\|_{\infty} \sup _{r \in\left[t_{i}, t_{i+1}\right]}\left|\mathcal{X}_{r, 0}(x)-\mathcal{X}_{t_{i}, 0}(x)\right| .
$$

For this, we have used the fact that $b$ is Lipschitz continuous and $1+\frac{T}{n}\|\nabla b\|_{\infty} \leq$ $\exp \left(T\|\nabla b\|_{\infty} / n\right)$.

It can be proved that similarly to the Brownian path, each path of $\mathcal{X}_{t, 0}(x)$ is $\alpha$ Hölder continuous for any $\alpha<1 / 2$. This is a direct consequence of the Kolmogorov lemma on the regularity of stochastic processes. This proves that the right hand side of (36) converges to 0 at rate $\alpha<1 / 2$.

\section{B The heat and the transport semi-group}

The underlying Banach space is $\mathrm{B}=\mathrm{C}_{\mathrm{z}}\left(\mathbb{R}^{d}, \mathbb{R}\right)$, the space of continuous, bounded functions that vanish at infinity. The norm on $\mathrm{B}$ is $\|f\|=\sup _{x \in \mathbb{R}^{d}}|f(x)|$.

\section{B.1 The heat semi-group}

The heat semi-group is

$$
P_{t} f(x)=\int \frac{1}{(2 \pi t)^{d / 2}} \exp \left(\frac{-|x-y|^{2}}{2 t}\right) f(y) \mathrm{d} y
$$

for a measurable function $g$ which is bounded or in $\mathrm{L}^{2}\left(\mathbb{R}^{d}\right)$, the space of square integrable functions.

Since the marginal distribution of the Brownian motion $B$ at any time $t$ is normal one with mean 0 and variance $t, P_{t} f(x)=\mathbb{E}\left[f\left(x+B_{t}\right)\right]$.

Using Fourier transform or computing derivatives,

$$
\partial_{t} P_{t} f(x)=\frac{1}{2} \triangle P_{t} f(x), x \in \mathbb{R}^{d}, t>0 .
$$

Multiplying the above equation by $g(x) \in \mathrm{C}_{\mathrm{c}}^{2}\left(\mathbb{R}^{d}, \mathbb{R}\right)$, performing an integration by parts then integrating between 0 and $t$ lead to

$$
\int_{\mathbb{R}^{d}}\left(P_{t} g(x)-g(x)\right) f(x) \mathrm{d} x=\frac{1}{2} \int_{0}^{t} \int_{\mathbb{R}^{d}} P_{t} f(x) \triangle g(x) \mathrm{d} x .
$$


Then, passing to the limit and since $f$ is freely chosen,

$$
\lim _{t \rightarrow 0} \frac{P_{t} g(x)-g(x)}{t}=\frac{1}{2} \triangle g(x), \forall x \in \mathbb{R}^{d}, g \in \mathrm{C}_{\mathrm{c}}^{2}\left(\mathbb{R}^{d}, \mathbb{R}\right) .
$$

Thus, if $(\mathfrak{L}, \operatorname{Dom}(\mathfrak{L}))$ is the infinitesimal generator of $\left(P_{t}\right)_{t \geq 0}$ (this is necessarily a close operator $), \mathfrak{L}=\frac{1}{2} \triangle$ on $\mathcal{C}_{\mathrm{c}}^{2}\left(\mathbb{R}^{d}, \mathbb{R}\right) \subset \operatorname{Dom}(\mathfrak{L})$. The latter space being dense in the underlying space $\mathrm{C}_{\mathrm{z}}\left(\mathbb{R}^{d}, \mathbb{R}\right)$ with respect to $\|\cdot\|_{\infty}$ and $\operatorname{Dom}(\mathfrak{L})$ with respect to the graph norm $\|\cdot\|_{\infty}+\|\mathfrak{L} \cdot\|_{\infty},(37)$ characterizes $(\mathfrak{L}, \operatorname{Dom}(\mathfrak{L}))$ when $\mathrm{C}_{\mathrm{z}}\left(\mathbb{R}^{d}, \mathbb{R}\right)$ is the ambient Banach space.

In other words, we recover that the infinitesimal generator of the Brownian motion is $\mathfrak{L}=\frac{1}{2} \triangle$ with a suitable domain. This could of course be easily obtained from the Itô's formula. Also, we see the link between the heat equation (B.1), the Brownian motion and the heat semi-group.

\section{B.2 The transport semi-group}

Let us consider now the flow $\left(\mathcal{Y}_{t}\right)$. Since $\mathcal{Y}(x)$ is solution to $\mathcal{Y}_{t}(x)=x+\int_{0}^{t} b\left(\mathcal{Y}_{s}(x)\right) \mathrm{d} s$, the Newton formula for $f \in \mathrm{C}_{\mathrm{c}}^{1}\left(\mathbb{R}^{d}, \mathbb{R}\right)$ implies that

$$
f\left(\mathcal{Y}_{t}(x)\right)-f(x)=\int_{0}^{t} b\left(\mathcal{Y}_{s}(x)\right) \nabla f\left(\mathcal{Y}_{s}(s)\right) \mathrm{d} s .
$$

Hence, the infinitesimal generator of $\left(Q_{t}\right)_{t \geq 0}$ is $\mathfrak{B}=b \nabla$. whose domain $\operatorname{Dom}(\mathfrak{B})$ is the closure of $\mathrm{C}_{\mathrm{c}}^{1}\left(\mathbb{R}^{d}, \mathbb{R}\right)$ to the graph norm (see e.g. [17, § II.3.28, p. 91]).

The semi-group $\left(Q_{t}\right)_{t>0}$ is also linked to a PDE, called the transport equation. We have seen in Proposition 2 that $x \mapsto \mathcal{Y}_{t}(x)$ is differentiable. It is actually of class $\mathrm{C}^{1}\left(\mathbb{R}^{d}, \mathbb{R}\right)$. Thus, $Q_{t} f(x)=f \circ \mathcal{Y}_{t}(x)$ is also differentiable. Applying the Newton formula to $f \circ \mathcal{Y}_{t}=Q_{t} f$ and using the flow property of $\mathcal{Y}_{t}$ leads to

$$
f\left(\mathcal{Y}_{t+\epsilon}(x)\right)-f\left(\mathcal{Y}_{t}(x)\right)=Q_{t} f\left(\mathcal{Y}_{\epsilon}(x)\right)-Q_{t} f(x)=\int_{0}^{\epsilon} b\left(\mathcal{Y}_{s}(x)\right) \nabla Q_{t} f\left(\mathcal{Y}_{s}(x)\right) \mathrm{d} s .
$$

Dividing each side by $\epsilon$ and passing to the limit implies that

$$
\partial_{t} Q_{t} f(x)=b(x) \nabla Q_{t} f(x) .
$$

Conversely, it is also possible to start from the transport equation $\partial_{t} u(t, x)=$ $b(x) \nabla u(t, x)$ to construct the flow $\mathcal{Y}$ through the so-called method of characteristics, that is to find the paths $\mathcal{Z}: \mathbb{R}_{+} \rightarrow \mathbb{R}^{d}$ such that $u\left(t, \mathcal{Z}_{t}\right)$ is constant over the time $t$.

Acknowledgement. I wish to thank K. Coulibaly-Pasquier, V. Bally and A. Kohatsu-Higa for some motivating and interesting discussions on this approach. This article is a follow-up of a talk given at the "groupe de travail" of the Probability and Statistics teams of Institut Élie Cartan de Lorraine (Nancy) on the link between the Trotter-Kato-Lie and the Feynman-Kac formula, and I am grateful to the audience for his/her patience. 


\section{References}

[1] J. Akahori, T. Amaba, and S. Uraguchi. "An algebraic approach to the Cameron-Martin-Maruyama-Girsanov formula". In: Math. J. Okayama Univ. 55 (2013), pp. 167-190.

[2] L. Ambrosio and D. Trevisan. Lecture notes on the DiPerna-Lions theory in abstract measure spaces. 2015. arXiv: 1505.05292.

[3] R. Bafico and P. Baldi. "Small random perturbations of Peano phenomena". In: Stochastics 6.3-4 (1981/82), pp. 279-292. DOI: 10.1080/17442508208833208.

[4] V. Bally and A. Kohatsu-Higa. "A probabilistic interpretation of the parametrix method". In: Ann. Appl. Probab. 25.6 (2015), pp. 3095-3138. DOI: 10.1214/ 14-AAP1068.

[5] V. Bally and C. Rey. "Approximation of Markov semigroups in total variation distance". In: Electron. J. Probab. 21 (2016), Paper No. 12, 44. DoI: 10.1214/16-EJP4079.

[6] D. R. Bell. The Malliavin calculus. Vol. 34. Pitman Monographs and Surveys in Pure and Applied Mathematics. Longman Scientific \& Technical, Harlow; John Wiley \& Sons, Inc., New York, 1987.

[7] A. Beskos and G. O. Roberts. "Exact simulation of diffusions". In: Ann. Appl. Probab. 15.4 (2005), pp. 2422-2444. DOI: 10.1214/105051605000000485.

[8] S. Blanes, F. Casas, and A. Murua. "Splitting and composition methods in the numerical integration of differential equations". In: Bol. Soc. Esp. Mat. Apl. S MA 45 (2008), pp. 89-145.

[9] R. H. Cameron and W. T. Martin. "The transformation of Wiener integrals by nonlinear transformations". In: Trans. Amer. Math. Soc. 66 (1949), pp. 253-283. DOI: 10.1090/S0002-9947-1949-0031196-6.

[10] R. H. Cameron and W. T. Martin. "Transformations of Wiener integrals under a general class of linear transformations". In: Trans. Amer. Math. Soc. 58 (1945), pp. 184-219.

[11] R. H. Cameron and W. T. Martin. "Transformations of Wiener integrals under translations". In: Ann. of Math. (2) 45 (1944), pp. 386-396.

[12] P. R. Chernoff. Product formulas, nonlinear semigroups, and addition of unbounded operators. Memoirs of the American Mathematical Society, No. 140. American Mathematical Society, Providence, R. I., 1974.

[13] R. J. DiPerna and P. L. Lions. "Ordinary differential equations, transport theory and Sobolev spaces". In: Inventiones Mathematicae 98.3 (1989), pp. 511547. 
[14] C. Doléans-Dade. "Quelques applications de la formule de changement de variables pour les semimartingales". In: Z. Wahrscheinlichkeitstheorie und Verw. Gebiete 16 (1970), pp. 181-194.

[15] J. L. Doob. "Conditional Brownian motion and the boundary limits of harmonic functions". In: Bull. Soc. Math. France 85 (1957), pp. 431-458.

[16] D. Duffie and P. Protter. "From Discrete- to Continuous-Time Finance: Weak Convergence of the Financial Gain Process". In: J. Math. Finance 2 (1992), pp. $1-15$.

[17] K.-J. Engel and R. Nagel. One-parameter semigroups for linear evolution equations. Vol. 194. Graduate Texts in Mathematics. With contributions by S. Brendle, M. Campiti, T. Hahn, G. Metafune, G. Nickel, D. Pallara, C. Perazzoli, A. Rhandi, S. Romanelli and R. Schnaubelt. Springer-Verlag, New York, 2000.

[18] M. Erraoui and E. H. Essaky. "Canonical representation for Gaussian processes". In: Séminaire de Probabilités XLII. Vol. 1979. Lecture Notes in Math. Springer, Berlin, 2009, pp. 365-381. DOI: 10.1007/978-3-642-01763-6_13.

[19] F. Flandoli. "Remarks on uniqueness and strong solutions to deterministic and stochastic differential equations". In: Metrika 69.2-3 (2009), pp. 101123. DOI: $10.1007 / \mathrm{s} 00184-008-0210-7$.

[20] I. V. Girsanov. "On transforming a class of stochastic processes by absolutely continuous substitution of measures". In: Theor. Probab. Appl. 5 (1960), pp. 285-301. DOI: $10.1137 / 1105027$.

[21] J. A. Goldstein. "Remarks on the Feynman-Kac formula". In: Partial differential equations and dynamical systems. Vol. 101. Res. Notes in Math. Pitman, Boston, MA, 1984, pp. 164-180.

[22] H. Gzyl. "The Feynman-Kac formula and the Hamilton-Jacobi equation". In: J. Math. Anal. Appl. 142.1 (1989), pp. 74-82. DOI: 10.1016/0022247X (89) 90165-0.

[23] E. Hairer, C. Lubich, and G. Wanner. Geometric numerical integration. 2nd ed. Vol. 31. Springer Series in Computational Mathematics. Structurepreserving algorithms for ordinary differential equations. Springer-Verlag, Berlin, 2006.

[24] H. Hult. "Approximating some Volterra type stochastic integrals with applications to parameter estimation". In: Stochastic Process. Appl. 105.1 (2003), pp. 1-32. DOI: 10.1016/S0304-4149(02)00250-8. 
[25] M. Jeanblanc, J. Pitman, and M. Yor. "The Feynman-Kac formula and decomposition of Brownian paths". In: Mat. Apl. Comput. 16.1 (1997), pp. 2752.

[26] M. Jeanblanc, M. Yor, and M. Chesney. Mathematical methods for financial markets. Springer Finance. Springer-Verlag London, Ltd., London, 2009. DOI: 10.1007/978-1-84628-737-4.

[27] M. Kac. "On distributions of certain Wiener functionals". In: Trans. Amer. Math. Soc. 65 (1949), pp. 1-13.

[28] T. Kato. "Trotter's product formula for an arbitrary pair of self-adjoint contraction semigroups". In: Topics in functional analysis (essays dedicated to M. G. Krein on the occasion of his 70th birthday). Vol. 3. Adv. in Math. Suppl. Stud. Academic Press, New York-London, 1978, pp. 185-195.

[29] A. Kohatsu-Higa, A. Lejay, and K. Yasuda. "Weak rate of convergence of the Euler-Maruyama scheme for stochastic differential equations with nonregular drift". In: Journal of Computational and Applied Mathematics 326C (2017), pp. 138-158. DOI: 10.1016/j.cam.2017.05.015.

[30] T. G. Kurtz and P. E. Protter. "Weak convergence of stochastic integrals and differential equations". In: Probabilistic models for nonlinear partial differential equations (Montecatini Terme, 1995). Vol. 1627. Lecture Notes in Math. Springer, Berlin, 1996, pp. 1-41. DOI: 10.1007/BFb0093176.

[31] R. Leandre. "Applications of the Malliavin calculus of Bismut type without probability". In: WSEAS Trans. Math. 5.11 (2006), pp. 1205-1210.

[32] S. Lie and F. Engel. Theorie der Transformationsgruppen. Teubner, Leipzig, 1888 .

[33] R. S. Liptser and A. N. Shiryaev. Statistics of random processes. I. General theory. 2nd ed. Vol. 5. Applications of Mathematics (New York). Translated from the 1974 Russian original by A. B. Aries. Springer-Verlag, Berlin, 2001. DOI: $10.1007 / 978-3-662-13043-8$.

[34] R. S. Liptser and A. N. Shiryaev. Statistics of random processes. II. Applications. 2nd ed. Vol. 6. Applications of Mathematics (New York). Translated from the 1974 Russian original by A. B. Aries. Springer-Verlag, Berlin, 2001. DOI: $10.1007 / 978-3-662-10028-8$.

[35] B. Maisonneuve. "Quelques martingales remarquables associées à une martingale continue". In: Publ. Inst. Statist. Univ. Paris 17.fasc. 3 (1968), pp. 1327. 
[36] P. Malliavin. Stochastic analysis. Vol. 313. Grundlehren der Mathematischen Wissenschaften. Springer-Verlag, Berlin, 1997. DOI: 10.1007/978-3-64215074-6.

[37] G. Maruyama. "On the transition probability functions of the Markov process". In: Nat. Sci. Rep. Ochanomizu Univ. 5 (1954), pp. 10-20.

[38] R. Mikulevičius and C. Zhang. "Weak Euler approximation for Itô diffusion and jump processes". In: Stoch. Anal. Appl. 33.3 (2015), pp. 549-571. DOI: 10.1080/07362994.2015.1014102.

[39] R. Mikulevičius and E. Platen. "Rate of convergence of the Euler approximation for diffusion processes". In: Math. Nachr. 151 (1991), pp. 233-239. DOI: $10.1002 / \mathrm{mana} .19911510114$.

[40] E. Nelson. "Feynman integrals and the Schrödinger equation". In: J. Mathematical Phys. 5 (1964), pp. 332-343.

[41] S. Ninomiya and N. Victoir. "Weak approximation of stochastic differential equations and application to derivative pricing". In: Appl. Math. Finance 15.1-2 (2008), pp. 107-121. DOI: 10.1080/13504860701413958.

[42] S. Orey. "Conditions for the absolute continuity of two diffusions". In: Trans. Amer. Math. Soc. 193 (1974), pp. 413-426.

[43] A. Pazy. Semigroups of linear operators and applications to partial differential equations. Vol. 44. Applied Mathematical Sciences. Springer-Verlag, New York, 1983. DOI: 10.1007/978-1-4612-5561-1.

[44] B. Roynette and M. Yor. Penalising Brownian paths. Vol. 1969. Lecture Notes in Mathematics. Springer-Verlag, Berlin, 2009. DOI: 10.1007/978-3540-89699-9.

[45] B. Simon. "Schrödinger semigroups". In: Bull. Amer. Math. Soc. (N.S.) 7.3 (1982), pp. 447-526. DOI: 10.1090/S0273-0979-1982-15041-8.

[46] A. V. Skorohod. "On the densities of probability measures in functional spaces". In: Proc. Fifth Berkeley Sympos. Math. Statist. and Probability (Berkeley, Calif., 1965/66). Univ. California Press, Berkeley, Calif., 1967, Vol. II: Contributions to Probability Theory, Part 1, Part 1.

[47] D. W. Stroock and S. R. S. Varadhan. Multidimensional diffusion processes. Classics in Mathematics. Reprint of the 1997 edition. Springer-Verlag, Berlin, 2006.

[48] S. Takanobu. "On the error estimate of the integral kernel for the Trotter product formula for Schrödinger operators". In: Ann. Probab. 25.4 (1997), pp. 1895-1952. DOI: $10.1214 / \mathrm{aop} / 1023481116$. 
[49] D. Talay. "Discrétisation d'une équation différentielle stochastique et calcul approché d'espérances de fonctionnelles de la solution". In: RAIRO Modél. Math. Anal. Numér. 20.1 (1986), pp. 141-179.

[50] H. F. Trotter. "On the product of semi-groups of operators". In: Proc. Amer. Math. Soc. 10 (1959), pp. 545-551.

[51] J. H. Van Schuppen and E. Wong. "Transformation of local martingales under a change of law". In: Ann. Probability 2 (1974), pp. 879-888.

[52] A. J. Veretennikov. "On strong solutions and explicit formulas for solutions of stochastic integral equations". In: Math. USSR Sb. 39.3 (1981), pp. 387403. DOI: 10.1070/SM1981v039n03ABEH001522.

[53] A. Zvonkin. "A transformation of the phase space of a diffusion process that removes the drift". In: Math. USSR Sb. 22 (1975), pp. 129-149. DOI: 10.1070/SM1974v022n01ABEH001689. 